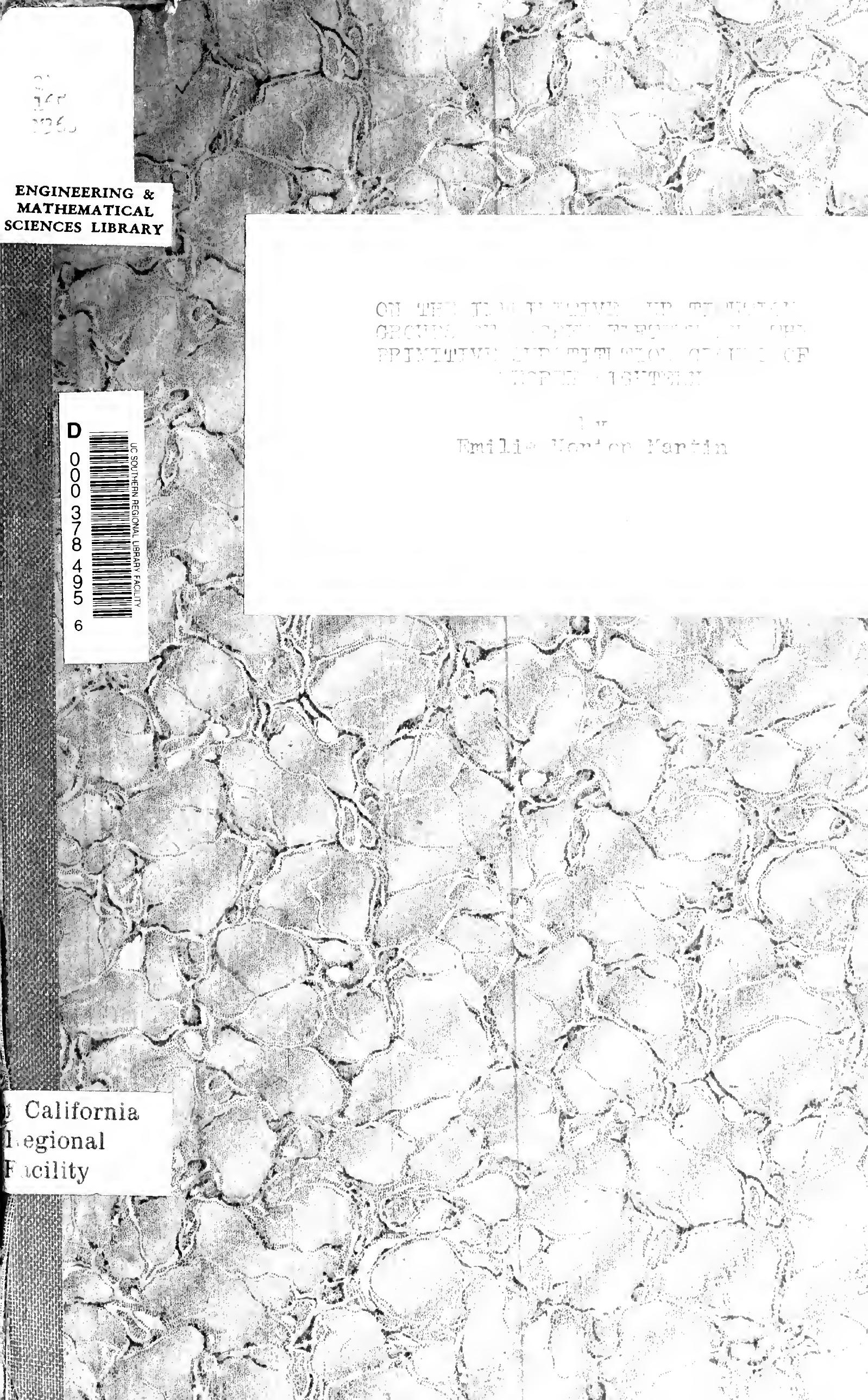




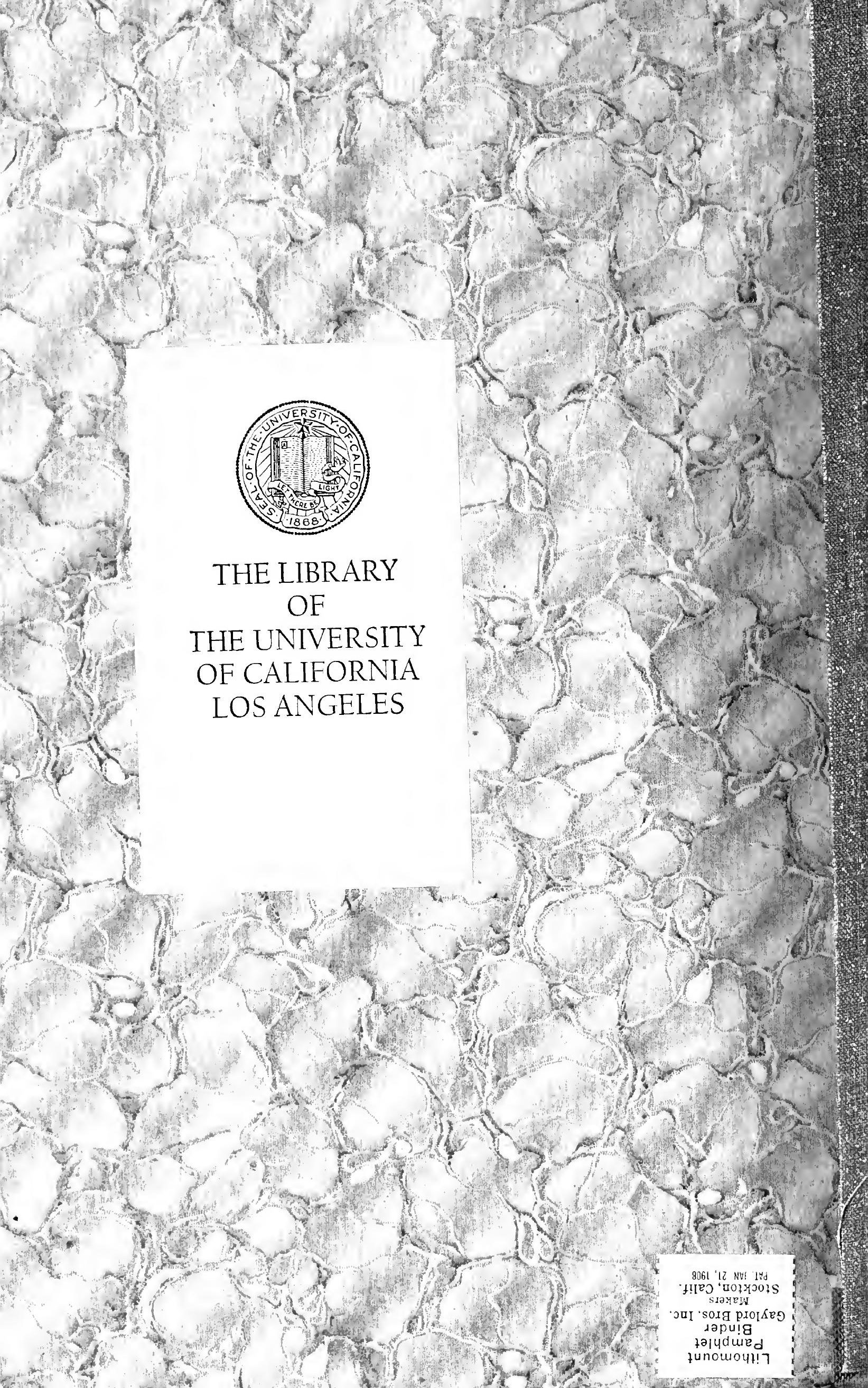




\title{
ON THE IMPRIMITIVE SUBSTITUTION GROUPS OF DEGREE FIFTEEN AND THE PRIMITIVE SUBSTITUTION GROUPS OF DEGREE EIGHTEEN
}

\author{
A DISSERTATION \\ PRESENTED TO THE FACULTY OF BRYN MAWR COLLEGE FOR THE DEGREE OF \\ DOCTOR OF PHILOSOPHY
}

BY EMILIE NORTON MARTIN

1901

EBe Eord Gaftimore (Preas

The Friedeniald Company

BALTIMOKE, MI., U. S. A. 


\section{ON THE IMPRIMITIVE SUBSTITUTION GROUPS OF DEGREE FIFTEEN AND THE PRIMITIVE SUBSTITUTION GROUPS OF DEGREE EIGHTEEN}

\section{A DISSERTATION}

PRESENTED TO THE FACULTY OF BRYN MAWR COLLEGE FOR THE DEGREE OF DOCTOR OF PHILOSOPHY

BY EMILIE NORTON MARTIN 
$\therefore \therefore \because \because \cdots$

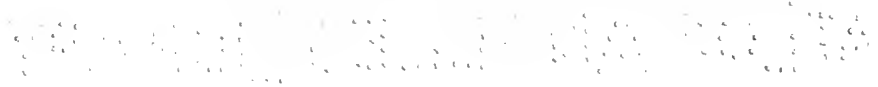




\title{
On the Imprimitive Substitution Groups of Degree Fifteen and the Primitive Substitution Groups of Degree Eighteen.
}

\author{
By Emilie Norton Martin.
}

The following work is, with some slight modifications, the same as that of which an abstract was presented at the summer meeting of the American Mathematical Society in 1899. With regard to the imprimitive groups of degree fifteen, which form the subject matter of the first part of this paper, it should be stated that I have added two new groups to the list as originally presented, namely, the groups with five systems of imprimitivity simply isomorphic to the alternating and symmetric groups of degree 5, and that Dr. Kuhn reported at the February meeting of the Society, 1900, that he had carried the investigation further, adding 28 to the 70 groups that I succeeded in finding.

In the second part of this paper the determination of the primitive groups of degree 18 depends to a great extent upon the lists of transitive groups of lower degrees already determined. Any new discovery of groups of degree less than 18 would necessitate an examination of such groups to determine whether they can be combined with others in such a way as to generate a primitive group of degree 18. This list, therefore, cannot claim to be absolutely complete, since omissions are always possible.

\section{Imprimitive Substitution Groups of Degree Fifteen.}

Every imprimitive group contains a self-conjugate intransitive subgroup consisting of all the operations that interchange the elements of the systems of imprimitivity among themselves without interchanging the systems. Therefore, the problem of the determination of all imprimitive groups of degree 15 falls into two parts: 1st, the determination of all intransitive groups of degree 15 
capable of becoming the self-conjugate subgroups of such imprimitive groups; $2 d$, the determination of substitutions that will interchange the systems of imprimitivity and at the same time fulfill other conditions depending upon the particular group under discussion. The intransitive self-conjugate subgroup is called for shortness the head, the remaining substitutions of the imprimitive group are designated as the tail, a terminology that has been adopted by Dr. G. A. Miller in his papers on imprimitive groups.

The elements of an imprimitive group of degree 15 may fall into three systems of five elements each, or into five systems of three each. For the first of these cases, certain theorems given by Dr. G. A. Miller (Quar. Jour. Math., vol. XXVIII, 1896) are useful. With a slight modification in notation in order to adapt them to the notation of this paper, they are as follows, where $G^{1}$ represents a group in the elements with index 1 , while $G^{2}$ and $G^{3}$ represent precisely the same group in the elements with indices 2 and 3.

Theorem I.-All the substitutions that can be used to construct tails are $\left(a_{1}^{1} a_{2}^{1} \ldots a_{n}^{1}\right)$ all $\left(a_{1}^{2} a_{2}^{2} \ldots a_{n}^{2}\right)$ all $\left(a_{1}^{3} a_{2}^{3} \ldots a_{n}^{3}\right)$ all $\left\{\left(a_{1}^{1} a_{1}^{2} a_{1}^{3}, a_{2}^{1} a_{2}^{2} a_{2}^{3} . \ldots . a_{n}^{1} a_{n}^{2} a_{n}^{3}\right),\left(a_{1}^{1} a_{1}^{2}, a_{2}^{1} a_{2}^{2}, \ldots . a_{n}^{1} a_{n}^{2}\right)\right\}$ $-\left(a_{1}^{1} a_{2}^{1} \ldots a_{n}^{1}\right)$ all $\left(a_{1}^{2} a_{2}^{2} \ldots a_{n}^{2}\right)$ all $\left(a_{1}^{3} a_{2}^{3} \ldots a_{n}^{3}\right)$ all.

THEOREM II.-If $G^{1}=\left(a_{1}^{1} a_{2}^{1} \ldots a_{n}^{1}\right)$ all, there are three imprimitive groups with the common head $\left(G^{1} G^{2} G^{3}\right)$ pos, and two with the common head $G^{1}$ pos $G^{2}$ pos $G^{3}$ pos $+G^{1}$ neg $G^{2}$ neg $G^{3}$ neg.

THEOREM III.-If $G^{1}=\left(a_{1}^{1} a_{2}^{1} \ldots a_{n}^{1}\right)$ pos, there are three imprimitive groups with the common head $G^{1} G^{2} G^{3}$, and three with the common head $\left(G^{1} G^{2} G^{3}\right)_{1,1,1}$.

THEOREM IV. - If the head is $G^{1} G^{2} G^{3}$, there is only one group which corresponds to $(a b c)$ cyc.

The possible heads for these groups are got either by the direct multiplication of transitive groups of degree 5 in the three systems of elements, or by the establishment of isomorphic relations between such groups.

The transitive groups of degree 5 are five in number, and fall naturally into two categories, the first containing the symmetric group and its self-conjugate subgroup, the alternating group, the second containing the metacyclic group, together with its two self-conjugate transitive subgroups. These five groups are represented respectively by

$$
\left(a_{1} a_{2} a_{3} a_{4} a_{5}\right) \text { all, }\left(a_{1} a_{2} a_{3} a_{4} a_{5}\right) \text { pos, }\left(a_{1} a_{2} a_{3} a_{4} a_{5}\right)_{20},\left(a_{1} a_{2} a_{3} a_{4} a_{5}\right)_{10},\left(a_{1} a_{2} a_{3} a_{4} a_{5}\right)_{5} .
$$


Fifteen and the Primitive Substitution Groups of Degree Eighteen.

From the first two groups come the following heads:

I. $\quad\left(a_{1}^{1} a_{2}^{1} a_{3}^{1} a_{4}^{1} a_{5}^{1}\right)$ all $\left(a_{1}^{2} a_{2}^{2} a_{3}^{2} a_{4}^{3} a_{5}^{2}\right)$ all $\left(a_{1}^{3} a_{2}^{3} a_{3}^{3} a_{4}^{3} a_{5}^{3}\right)$ all $=H_{1728000}$.

II. $\left\{\left(a_{1}^{1} a_{2}^{1} a_{3}^{1} a_{4}^{1} a_{5}^{1}\right)\right.$ all $\left(a_{1}^{2} a_{2}^{3} a_{3}^{2} a_{4}^{2} a_{5}^{2}\right)$ all $\left(a_{1}^{3} a_{2}^{3} a_{5}^{3} a_{4}^{3} a_{5}^{3}\right)$ all $\}$ pos $=H_{804000}$.

III. $\quad\left(a_{1}^{1} a_{2}^{1} a_{3}^{1} a_{4}^{1} \alpha_{5}^{1}\right) \operatorname{pos}\left(a_{1}^{2} a_{2}^{2} a_{3}^{2} a_{4}^{2} a_{5}^{2}\right) \operatorname{pos}\left(a_{1}^{3} a_{2}^{3} a_{3}^{3} a_{4}^{3} a_{5}^{3}\right)$ pos $+\left(a_{1}^{1} a_{2}^{1} a_{3}^{1} a_{1}^{1} a_{5}^{1}\right)$ neg $\left(a_{1}^{3} a_{2}^{2} a_{3}^{2} a_{4}^{2} a_{5}^{2}\right)$ neg $\left(a_{1}^{3} a_{2}^{3} a_{3}^{3} a_{4}^{3} a_{5}^{3}\right)$ neg $=I I_{4320000}$.

IV. $\quad\left(a_{1}^{1} a_{2}^{1} a_{3}^{1} a_{4}^{1} a_{5}^{1}\right)$ pos $\left(a_{1}^{2} a_{2}^{2} a_{3}^{2} a_{1}^{2} a_{5}^{2}\right) \operatorname{pos}\left(a_{1}^{3} a_{2}^{3} a_{3}^{3} a_{4}^{3} a_{5}^{3}\right) \operatorname{pos}=I I_{216000}$.

V. $\quad\left(a_{1}^{1} a_{2}^{1} a_{3}^{1} a_{4}^{1} a_{5}^{1} \cdot a_{1}^{2} a_{3}^{2} a_{3}^{3} a_{4}^{2} a_{5}^{2} \cdot a_{1}^{3} a_{2}^{3} a_{3}^{3} a_{4}^{3} e_{5}^{3}\right)$ all $=H_{120}$.

VI. $\quad\left(a_{1}^{1} a_{2}^{1} a_{3}^{1} a_{4}^{1} a_{5}^{1} . a_{1}^{2} a_{2}^{2} a_{3}^{2} a_{4}^{2} a_{5}^{2} \cdot a_{1}^{3} a_{2}^{3} a_{3}^{3} a_{4}^{3} a_{5}^{3}\right)$ pos $=H_{60}$.

From the three remaining groups come the heads:

VII. $\quad\left(a_{1}^{1} a_{2}^{1} a_{3}^{1} a_{4}^{1} a_{5}^{1}\right)_{20}\left(a_{1}^{3} a_{2}^{2} a_{3}^{3} a_{4}^{2} a_{5}^{2}\right)_{20}\left(a_{1}^{3} a_{2}^{3} a_{3}^{3} a_{4}^{3} a_{5}^{3}\right)_{20}=H_{8000}$.

VIII. $\quad\left\{\left(a_{1}^{1} a_{2}^{1} a_{3}^{1} a_{4}^{1} a_{5}^{1}\right)_{20}\left(a_{1}^{2} a_{2}^{2} r_{3}^{2} a_{4}^{2} a_{5}^{2}\right)_{: 0}\left(a_{1}^{3} a_{2}^{3} c_{3}^{3} c_{4}^{3} a_{5}^{3}\right)_{: 0}\right\}$ pos $=H_{4000}$.

IX. $\quad\left\{\left(a_{1}^{1} a_{2}^{1} a_{3}^{1} a_{4}^{1} a_{5}^{1}\right)_{20}\left(a_{1}^{2} a_{2}^{3} a_{3}^{2} a_{4}^{2} a_{5}^{2}\right)_{20}\left(a_{1}^{3} a_{2}^{3} a_{3}^{3} u_{4}^{3} a_{5}^{3}\right)_{20}\right\}_{10,10,10}=H_{20000}$.

X. $\quad\left(a_{1}^{1} a_{2}^{1} a_{3}^{1} a_{4}^{1} a_{5}^{1}\right)_{10}\left(a_{1}^{2} a_{2}^{3} a_{3}^{2} a_{4}^{3} a_{5}^{2}\right)_{10}\left(a_{1}^{3} a_{2}^{3} a_{3}^{3} a_{4}^{3} a_{5}^{3}\right)_{10}=H_{1000}$.

XI. $\quad\left\{\left(a_{1}^{1} a_{2}^{1} a_{3}^{1} a_{4}^{1} a_{5}^{1}\right)_{20}\left(a_{1}^{2} a_{2}^{2} a_{3}^{2} a_{4}^{2} a_{5}^{2}\right)_{20}\left(a_{1}^{3} a_{2}^{3} a_{3}^{3} a_{4}^{3} a_{5}^{3}\right)_{20}\right\}_{5,5,5}=I_{500}$.

XII. $\quad\left\{\left(a_{1}^{1} a_{2}^{1} a_{3}^{1} a_{4}^{1} a_{5}^{1}\right)_{10}\left(a_{1}^{2} e_{5}^{2} e_{3}^{3} a_{4}^{3} a_{5}^{2}\right)_{10}\left(a_{1}^{3} a_{2}^{3} a_{3}^{3} a_{4}^{3} a_{5}^{3}\right)_{10}\right\}_{5,5,5}=\Pi_{250}$.

XIII. $\quad\left(a_{1}^{1} a_{2}^{1} a_{3}^{1} a_{4}^{1} a_{5}^{1}\right)$ cyc $\left(a_{1}^{2} a_{2}^{2} a_{3}^{2} a_{4}^{2} a_{5}^{2}\right)$ cyc $\left(a_{1}^{3} a_{2}^{3} a_{3}^{3} a_{4}^{3} a_{5}^{3}\right)$ cyc $=H_{125}$.

XIV. $\quad\left(a_{1}^{1} a_{2}^{1} a_{3}^{1} a_{4}^{1} a_{5}^{1} \cdot a_{1}^{3} a_{2}^{2} a_{3}^{3} a_{4}^{2} a_{5}^{2} \cdot a_{1}^{3} a_{2}^{3} a_{3}^{3} a_{4}^{3} a_{5}^{3}\right)_{20}=H_{30}$.

XV. $\quad\left(a_{1}^{1} a_{2}^{1} a_{3}^{1} a_{4}^{1} a_{5}^{1} \cdot a_{1}^{2} a_{2}^{2} a_{3}^{2} a_{4}^{2} a_{5}^{2} \cdot a_{1}^{3} a_{2}^{3} e_{3}^{3} a_{4}^{3} a_{5}^{3}\right)_{10}=H_{10}$.

XVI. $\quad\left(a_{1}^{1} a_{2}^{1} a_{3}^{1} a_{4}^{1} a_{5}^{1} \cdot a_{1}^{2} a_{2}^{2} e_{3}^{2} a_{4}^{2} a_{5}^{2} \cdot a_{1}^{3} a_{5}^{3} a_{3}^{3} a_{4}^{3} a_{5}^{3}\right)$ cyc. $=\Pi_{5}$.

The groups corresponding to these heads may be isomorphic either to $\left(a^{1} c^{3} c^{3}\right)$ cyc or to $\left(a^{1} a^{2} a^{3}\right)$ all. To generate a group isomorphic to $\left(a^{1} a^{2} a^{3}\right)$ cyc a substitution with the following properties must be added to the head: it must have its cube in the head, it must interchange all three systems, and it must transform the head into itself. Calling the group so found $G$, the groups isomorphic to $\left(a^{1} a^{2} a^{3}\right)$ all may be found by combining with $G$ any substitution that has its square in the head, that interchanges two of the systems leaving the third unaffected, and that transforms $H$ into itself, and $G$ into itself.

As all the heads given above are symmetric in the three sets of elements, each head furnishes two groups by means of the symmetrically formed substitutions $s=a_{1}^{1} a_{1}^{3} a_{1}^{3} \cdot a_{2}^{1} a_{2}^{2} a_{2}^{3} \cdot a_{3}^{1} a_{3}^{2} a_{3}^{3} \cdot a_{4}^{1} a_{4}^{*} a_{4}^{3} \cdot a_{5}^{1} a_{5}^{2} a_{5}^{3}, t=a_{1}^{1} a_{1}^{2} \cdot a_{2}^{1} a_{2}^{2} \cdot a_{3}^{1} a_{3}^{2} \cdot a_{4}^{1} a_{4}^{2} \cdot a_{5}^{1} a_{5}^{2}$.

The letters $s$ and $t$ are used throughout this section of the paper to denote these particular substitutions, other substitutions fulfilling the same conditions being denoted by the same letters with suffixes. 
According to Theorem I, any $s_{a}$ or $t_{a}$ must be the product of some substitution, $\sigma_{a}$, of the most general head, $I_{1728900}$, by $s$ or $t$. Therefore $\sigma_{a}$ nulust be a substitution of a subgroup of $H_{1728000}$ that contains the special $H$ under consideration as a selfconjugate subgroup.

We may now proceed to the determination of the groups to be derived from the various heads taken in order.

I. $H_{1728000}$ gives us, according to Theorem I, only the two groups,

$$
\text { and }\left\{H_{1 ; 28000}, s, t\right\} \text { of order } 10368000 .
$$

II. $H_{861000}$ gives us, in accordance with Theorem II, three distinct groups. of these, two are the groups,

$$
\begin{aligned}
& \left\{H_{\varepsilon 61000}, s\right\} \text { of order } 2592000_{1}, \\
& \left\{H_{861000}, \varepsilon, t\right\} \text { of order } 5184000_{2},
\end{aligned}
$$

A $\sigma$ that transforms the head into itself without belonging in the head is $\sigma=a_{1}^{\frac{1}{1}} a_{2}^{1}$. This cannot be combined with $s$, as $(\sigma s)^{3}$ is an odd substitution ; it may, however, be combined with $t$. The remaining group is therefore

$$
\left\{H_{864000}, s, a_{1}^{1} a_{: 2}^{1} \cdot t_{\}}^{\prime} \text { of order } 5184000_{3}\right. \text {. }
$$

Of these two groups of order 5184000 , the first contains both odd and even substitutions, the second only even.

III. $H_{13: 0: 0}$ gives, by Theorem II, the two groups

$$
\begin{aligned}
& \left\{H_{43: 000}, s\right\} \text { of order } 1296000_{1}, \\
& \left\{H_{432000}, s, t_{\}}^{\prime} \text { of order } 2592000_{2} .\right.
\end{aligned}
$$

IV. $H_{216000}$ gives us, by Theorem III, three distinct groups. $\sigma=a_{1}^{1} a_{2}^{1}$ transforms the head iuto itself, but when combined with $s$ it gives an odd substitution whose cube cannot be found in the head. The substitution $\sigma t$ furnishes us howerer, with a new $t_{a}$. The three groups are, therefore,

$$
\begin{aligned}
& \left\{H_{216010}, s ; \text { of order } 648000,\right. \\
& \left\{H_{216010}, s, t\right\} \text { of order } 1296000_{2}, \\
& \left\{H_{216000}, s, a_{1}^{1} \alpha_{2}^{1} . t\right\} \text { of order } 1296000_{3} .
\end{aligned}
$$

The two groups of order 1296000 are distinct, since the one contains both odd and even substitutions, the other only even. 
Fifteen and the Primitive Suldstitution Groups of Degree Eighteen.

V. $\Pi_{120}$ is not contained self-conjugately in any larger subgroup of $I_{17: 8000}$, therefore only the two following groups can be formed from it:

$$
\begin{aligned}
& \left\{H_{120}, s\right\} \text { of order } 360_{1}, \\
& \left\{H_{120}, s, t\right\} \text { of order } 720 .
\end{aligned}
$$

VI. $I_{60}$ gives, in accordance with Theorem III, three groups:

$$
\begin{aligned}
& \left\{H_{60}, s\right\} \text { of order } 180, \\
& \left\{H_{60}, s, t\right\} \text { of order } 360_{2}, \\
& \left\{H_{60}, s, a_{1}^{1} a_{2}^{1} \cdot a_{1}^{2} a_{2}^{2} \cdot a_{1}^{3} \alpha_{2}^{3} . t_{\}}^{\prime} \text { of order } 360_{3} .\right.
\end{aligned}
$$

The last of these groups consists entirely of even substitutions.

The remaining heads are all composed of substitutions of the type

$$
v_{a^{1}}^{a} u_{a^{1}}^{a^{\prime}} v_{a^{2}}^{\beta} u u_{u^{2}}^{\beta^{\prime}} v_{u^{3}}^{\gamma} u u_{a^{3}}^{\gamma^{\prime}},
$$

where $v_{a^{1}}=a_{2^{2}}^{1} u_{3}^{1} a_{5}^{1} a_{4}^{1}, u_{a^{2}}=a_{1}^{1} a_{2}^{1} a_{3}^{1} a_{t^{1}}^{1} t_{5}^{1}$, while $v_{a^{2}}, u_{a^{2}}, v_{a^{3}}, u_{a^{3}}$ denote the same substitutions written in elements with the indices 2 and 3 respectively. The substitutions $v_{a^{1}}, u_{a^{1}}$ generate the metacyclic group in the five elements with index 1 , these substitutions being subject to the conditions

$$
v_{a^{1}}^{4}=1, \quad u_{a^{1}}^{5}=1, \quad u_{a^{1}}^{x} v_{u^{1}}^{y}=v_{a^{1}}^{y} u_{a^{1}}^{2^{\prime \prime} x} .
$$

The most general $s_{a}$ is given by

From this we find

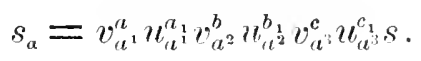

$$
s_{a}^{i 3}=v_{a^{2}}^{a+b+c} u_{a^{2}}^{\lambda} v_{a^{2}}^{a+b+c} u_{a^{2}}^{\mu} v_{a^{2}}^{a+b+c} u_{a^{2}}^{v},
$$

where

$$
\left.\begin{array}{l}
\lambda=2^{c}\left(2^{\prime} a_{1}+b_{1}\right)+c_{1}, \\
\mu=2^{\prime \prime}\left(2^{c} b_{1}+c_{1}\right)+a_{1}, \\
\nu=2^{\prime \prime}\left(2^{n} c_{1}+a_{1}\right)+b_{1} .
\end{array}\right\}
$$

Transformation of the general substitution (1) by $s_{a}$ gives us

$$
s_{a}^{-1} v_{a^{1}}^{\alpha} u_{a^{1}}^{\alpha^{\prime}} v_{a^{2}}^{\beta} u_{a^{2}}^{\beta^{\prime}} v_{a^{3}}^{\gamma} u_{a^{3}}^{\gamma} s_{a}=v_{a^{1}}^{\gamma} u_{a^{2}}^{v^{\prime}} v_{a^{2}}^{a} u_{a^{2}}^{\lambda} v_{a^{3}}^{\beta} u_{a^{3}}^{\mu} \text {, }
$$

where

$$
\left.\begin{array}{l}
\lambda=a_{1}+2^{a} \alpha^{\prime}-2^{a} a_{1}, \\
\mu=b_{1}+2^{b} \beta^{\prime}-2^{\beta} b_{1}, \\
\nu=c_{1}+2^{c} \gamma^{\prime}-2^{\gamma} c_{1},
\end{array}\right\}
$$

The general substitution of the group $G=\left\{I /, s_{a}\right\}$ is

$$
T=s_{a}^{r} v_{a^{2}}^{a} u_{a^{2}}^{a^{\prime}} v_{a^{2}}^{\beta} u_{a^{2}}^{\beta} v_{a^{3}}^{\gamma} n_{a^{3}}^{\gamma^{\prime}} .
$$


The most general $t_{\beta}$ is given by

$$
t_{\beta}=v_{a^{1}}^{a_{2}} u_{a^{3}}^{a_{3}} v_{a^{2}}^{b_{2}} u_{a^{3}}^{b_{3}} v_{a^{3}}^{c_{9}} u_{a^{3}}^{c_{3}} t .
$$

Upon squaring this substitution, we get

$$
t_{\beta}^{2}=v_{a^{1}}^{a_{2}+b_{2}} u_{a^{1}}^{\lambda} v_{a^{2}}^{a_{3}}+b_{2} u_{a^{2}}^{\mu} v_{a^{3}}^{2 c_{2}} u_{a^{3}}^{\nu},
$$

where

$$
\left.\begin{array}{l}
\lambda=2^{b_{2}} a_{3}+b_{3}, \\
\mu=2^{a_{2}} b_{3}+a_{3}, \\
v^{\prime}=2^{c_{2}} c_{3}+c_{3} .
\end{array}\right\}
$$

On transforming the general substitution $T$ by the general $t_{\beta}$, we have, after a straight-forward calculation, the following expression for the case $x=1$ :

$$
t_{\beta}^{-1} T_{x=1} t_{\beta}=s^{2} v_{u^{2}}^{-a_{2}+b_{2}+\beta+a} u_{a^{1}}^{\lambda} v_{a^{2}}^{a_{2}}-c_{2}+a+{ }^{c} u_{a^{2}}^{\mu} v_{a^{3}}^{-b_{2}}+c_{2}+\gamma+{ }^{b} u_{a_{3}}^{v},
$$

where

$$
\left.\begin{array}{l}
\lambda=-2^{h_{2}-a_{2}+\beta+a} a_{3}+2^{\beta+b_{2}} a_{1}+2^{b_{2}} \beta^{\prime}+b_{3}, \\
\mu=-2^{\alpha_{2}-c_{2}+a+c} c_{3}+2^{a+a_{2}} c_{1}+2^{a_{2}} \alpha^{\prime}+a_{3}, \\
\nu^{\prime}=-2^{c_{2}-b_{2}+\gamma+b} b_{3}+2^{\gamma+c_{2}} b_{1}+2^{c_{2}} \gamma^{\prime}+c_{3} .
\end{array}\right\}
$$

We may now return to the consideration of special groups.

VII. $H_{\text {zon }}$ gives only the two groups formed with $s$ and $t$, as any $\sigma$ that might be used is already contained in this head. The groups are, therefore,

$$
\left\{\begin{array}{l}
\left\{H_{8000}, s\right\} \text { of order } 24000_{1}, \\
\left\{H_{8000}, s, t_{\}}^{\prime} \text { of order } 48000 .\right.
\end{array}\right.
$$

VIII. $\Pi_{4000}$ has the general substitution (1) subject to the condition $\alpha+\beta$ $+\gamma \equiv 0(\bmod 2)$. From $(3)$, it is evident that $s_{a}$ is subject to the condition $a+b$ $+c \equiv 0(\bmod 2)$. Therefore, $s_{a}$ is already in the group generated by $H_{4000}$ and by $s$, and there is only one group isomorphic to $\left(a^{1} a^{2} a^{3}\right)$ cyc. We find by (9) that every $t_{\beta}$ has its square in the head, and by (11), that every $t_{\beta}$ transforms the head into itself, therefore, we may take as a new $t_{\beta}$ the simplest substitution for which $\iota_{2}+b_{2}+c_{2} \equiv 1(\bmod 2)$, viz. :

$$
v_{a} t=a_{1}^{1} a_{1}^{2} \cdot a_{2}^{1} \alpha_{3}^{2} a_{3}^{1} a_{5}^{2} \alpha_{5}^{1} \alpha_{4}^{2} a_{1}^{1} a_{2}^{2} .
$$

The three groups with this head are, therefore,

$$
\begin{aligned}
& \left\{H_{4000}, s\right\} \text { of order } 12000_{1}, \\
& \left\{H_{4000}, s, t\right\} \text { of order } 24000_{2}, \\
& \left\{H_{4000}, s, v_{a} t_{;}^{\prime} \text { of order } 24000_{3} .\right.
\end{aligned}
$$


Of these groups the first and third consist of even substitutions, the second of even and odd.

IX. $\quad H_{2000}$ has the general substitution subject to the condition $\alpha \equiv \beta \equiv \gamma(\bmod$ 2). From (3) and (5), it is plain that every $s_{a}$ can be used to generate a group of the kind required. The only possible form for the cofactor of $s$, if it is not to give the group generated by $s$ and the head, is $v_{a^{2}}^{a} v_{a^{2}}^{b} v_{a^{3}}^{e}$, where $a, b, c$ do not fulfill the condition $a \equiv b \equiv c(\bmod 2)$. The simplest form for such a cofactor, and a form to which all others reduce, is found by making two of the exponents vanish and the third become equal to 1 , e. g., $s_{1}=v_{a^{1}} s=a_{1}^{1} a_{1}^{2} a_{1}^{3}, a_{2}^{1} a_{3}^{3} a_{3}^{3} a_{3}^{1} a_{5}^{2} a_{5}^{3} a_{5}^{1} a_{4}^{2} a_{4}^{3} a_{4}^{1} a_{2}^{2} a_{2}^{3}$. Now, $s_{1}^{4}=s_{1} \cdot v_{a^{2}} v_{a^{2}} v_{a^{3}}$ and $s_{1}^{8}=s_{1}^{2} \cdot v_{a^{2}}^{2} v_{a^{2}}^{2} v_{a^{3}}^{2}$; we may, therefore, take $s_{1}^{4}$ as the $s$. in the place of $s_{1}$ and still have the same group. But $s_{1}^{4}=\left(v_{a^{2}}^{2} u_{u^{3}}^{3}\right)^{-1} s\left(v_{a^{2}}^{2} v_{a^{3}}^{3}\right)_{a}$ therefore the group we have now found is merely the transformed of the group generated by $s$ with respect to the substitution $v_{a^{2}}^{2} v_{a^{3}}^{3}$. Consequently, there is but one group corresponding to the cyclic group of degree three.

If, in addition to the group given by $t$, we have a group given by $t_{\beta}$, then according to the relations derived from $(11), a_{2} \equiv b_{2} \equiv c_{2}(\bmod 2)$, i. e., the possible values of $t_{\beta}$ are already present in the group generated with the help of $t$. The two imprimitive groups with this head are, therefore, the groups

$$
\begin{aligned}
& \left\{H_{2000}, s\right\} \text { of order } 6000 . \\
& \left\{H_{2000}, s, t\right\} \text { of order } 12000_{2} .
\end{aligned}
$$

In this, and all following work, the terms $u$ in the cofactors of $s$ and $t$ are taken as unity, unless the contrary is expressly stated.

X. $I_{1000}$ has its general substitution subject to the condition $\alpha \equiv \beta \equiv \gamma \equiv 0$ (mod 2). By Theorem IV, this head gives only one group isomorphic to $(a b c)$ cyc. If, in addition to the substitution $t$, there is a substitution $t_{\beta}$, the relations satisfied by the exponents of the $v^{\prime} \mathrm{s}$ in (11) reduces to $a_{2} \equiv b_{2} \equiv c_{2}$ (mod 2). We have, therefore, two distinct groups according as $a_{2}$ is even or odd. The three groups with this head are

$$
\begin{aligned}
& \left\{H_{1000}, s\right\} \text { of order } 3000_{1}, \\
& \left\{H_{1000}, s, t\right\} \text { of order } 6000_{2}, \\
& \left\{\Pi_{1000}, s, v_{a^{2}} v_{a^{2}} v_{a^{3}} t\right\} \text { of order } 6000_{3} .
\end{aligned}
$$

XI. $\Pi_{500}$ subjects the general substitution to the conditions $\alpha=\beta=\gamma$, where $\alpha=0,1,2,3$. Since every substitution $s_{\alpha}$ satisfies the necessary conditions, the following independent types of $s_{a}$ must be examined: $v_{a^{1}} s, v_{a^{1}}^{2} s, v_{a^{1}}^{3} s, v_{a^{2}} v_{a^{2}}^{2} s$. The 
fourth power of these substitutions is in every case the transformed of $s$ with respect to some combination of the $v$ 's; therefore, they give nothing new. The possible forms for $t_{\beta}$ are derived from the equation easily deducible from (11); $-a_{2}+b_{2} \equiv a_{2}-\cdots c_{2} \equiv-b_{2}+c_{2}(\bmod 4)$, which, taken in conjunction with the limited range of values of $a_{2}, b_{2}, c_{2}$, gives $a_{2}=b_{2}=c_{2}$. That is, every possible $t_{\beta}$ is already included in the group generated by $t$. This head gives accordingly only the two groups,

$$
\begin{aligned}
& \left\{H_{500}, s\right\} \text { of order } 1500_{1}, \\
& \left\{\Pi_{500}, s, t\right\} \text { of order } 3000_{2} .
\end{aligned}
$$

XII. $H_{250}$ subjects the general substitution (1) to the conditions $a=\beta=\gamma \equiv 0$ (mod 2). To determine an $s_{\alpha}$, we have from $(3)$ the condition $a+b+c \equiv 0$ $(\bmod 2)$. An examination of the four apparently distinct types of $s_{a}, v_{1}^{2} s, v_{1} v_{z} s$, $v_{1}^{3} v_{2} s, v_{1}^{3} v_{2}^{3} s$, shows that just as in the last set of groups, these each give a group that can be derived from the group generated by $s$ by means of an easy transformation.

The possible forms $t_{\beta}$ must fulfill the conditions, deducible from (11), $-a_{2}+$ $b_{2} \equiv-b_{2}+c_{2} \equiv-c_{2}+a_{2} \equiv 0(\bmod 2)$ and also $-a_{2}+b_{2} \equiv a_{2}-c_{2}(\bmod 4)$. These reduce to the simple condition $a_{2}=b_{2}=c_{2}$, which furnishes the substitution $t_{\beta}=v_{a^{2}} v_{a^{2}} v_{a^{3}} t$. This head gives therefore the three groups,

$$
\begin{aligned}
& \left\{H_{250}, s\right\} \text { of order } 750_{1}, \\
& \left\{H_{250}, s, t\right\} \text { of order } 1500_{2}, \\
& \left\{H_{250}, s, v_{a^{2}} v_{2^{2}} v_{a^{3}} t\right\} \text { of order } 1500_{3} .
\end{aligned}
$$

The second group alone contains odd substitutions.

XIII. $H_{125}$ gives in accordance with Theorem IV only one group in which the systems are interchanged cyclically. The general substitution of this head is subject to the condition $\alpha=\beta=\gamma=0$. Applying this condition to (9) and (11) we find $a_{2}=b_{2}=c_{2}$, while $a_{2}$ lies under the further restriction of being even. Therefore we have in addition to $t$ the substitution,

$$
t_{\beta}=v_{a^{2}}^{2} v_{a^{2}}^{2} v_{a^{3}}^{2} t=a_{1}^{1} a_{1}^{2} \cdot a_{2}^{1} a_{5}^{2} \cdot a_{3}^{1} a_{4}^{2} \cdot a_{4}^{1} a_{3}^{2} \cdot a_{5}^{1} a_{2}^{2} \cdot a_{2}^{3} a_{5}^{3} \cdot a_{3}^{3} a_{4}^{3} .
$$

The three groups given by this head are,

$$
\begin{aligned}
& \left\{H_{125}, s ; \text { of order } 375,\right. \\
& \left\{\Pi_{125}, s, t\right\} \text { of order } 750_{2}, \\
& \left\{H_{125}, s, v_{a^{2}}^{2} v_{a^{2}}^{2} v_{a^{3}}^{2} t\right\} \text { of order } 750_{3} .
\end{aligned}
$$

XIV. $H_{20}$ imposes upon the exponents of the general term the conditions 
$\alpha=\beta=\gamma, \alpha^{\prime}=\beta^{\prime}=\gamma^{\prime}$. Making use of this in (5) and (6) we find $2^{a} \alpha^{\prime} \equiv$ $2^{b} \alpha^{\prime} \equiv 2^{c} \alpha^{\prime}(\bmod 5)$, which gives at once $a=b=c$. Using this latter equality in the equations that are deduced from (3) and (4) we find $a_{1}=b_{1}=c_{1}$ with the single exception of the case $a=0$, where the equations become indeterminate, being satisfied by every value of $a_{1}, b_{1}, c_{1}$. An examination of all of the apparently independent sets of value for $a_{1}, b_{1}, c_{1}$ shows that in every case the group is transformable into that generated by $s$ alone. In order to determine all substitutions $\mathrm{t}_{\beta}$ we use the equation, derived from (11), $-a_{2}+b_{2} \equiv a_{2}-c_{2} \equiv c_{2}-b_{2}$ (mod. 4), from which follows at once $a_{2}=b_{2}=c_{2}$. From (12), by making use of the special case $\alpha=\beta=\gamma=0$, can be derived the relations $-a_{3}+b_{3} \equiv-c_{3}$ $+a_{3} \equiv-b+c_{3}(\bmod .5) ; \mathrm{i}, \mathrm{e}, a_{3}=b_{3}=c_{3}$. The only groups with this head are therefore the two groups,

$$
\left\{\begin{array}{l}
\left\{H_{20}, s\right\} \text { of order } 60_{1}, \\
\left\{\Pi_{20}, s, t\right\} \text { of order } 120 .
\end{array}\right.
$$

XV. $\quad H_{10}$ has the general term (1) subject to the conditions $\alpha=\beta=\gamma \equiv 0$ $(\bmod 2), \alpha^{\prime}=\beta^{\prime}=\gamma^{\prime}$. By precisely the same line of argument as that laid down in the preceding case we arrive at the conclusion $a=b=c, a_{1}=b_{1}=c_{1}, a_{2}=$ $b_{2}=c_{2}, a_{3}=b_{3}=c_{3}$. In this work, too, the indeterminate values of $a_{1}, b_{1}, c_{1}$ require a careful examination that leads to no new group. From this head come, therefore, the three groups,

$$
\begin{aligned}
& \left\{\Pi_{10}, s\right\} \text { of order } 30_{1}, \\
& \left\{\Pi_{10}, s, t\right\} \text { of order } 60_{2}, \\
& \left\{H_{10}, s, v_{t^{2}} v_{a^{2}} v_{a^{3}} t\right\} \text { of order } 60_{3} .
\end{aligned}
$$

Of these three groups the second alone involves odd substitutions.

XVI. $\quad H_{5}$ imposes upon the general term the conditions $\alpha=\beta=\gamma=0, \alpha^{\prime}=$ $\beta^{\prime}=\gamma^{\prime}$, By arguments similar to those used in the last two cases, with the further addition of the condition imposed by $(3), a+b+c \equiv 0(\bmod 4)$, we find $a=b=c=0, a_{1}=b_{1}=c_{1}$. In the determination of $t_{\beta}$ we see at once from (9) that $c_{2}$ must be even, while from (11) we find $a_{2}=b_{2}=c_{2}$, and from (12) $a_{3}=b_{3}=c_{3}$.

The groups given by this head are as follows:

$$
\begin{aligned}
& \left\{H_{5}, s\right\} \text { of order } 15, \\
& \left\{H_{5}, s, t\right\} \text { of order } 30_{2}, \\
& \left\{H_{5}, s, v_{a^{1}}^{2} v_{a^{2}}^{2} v_{a^{3}}^{2} t\right\} \text { of order } 30_{3} .
\end{aligned}
$$


Passing now to the case of five systems of three elements each, there are seven heads considered in this paper, six involving all the systems symmetrically, the remaining head being unity.

I. $\left(a_{1}^{1} a_{2}^{1} a_{3}^{1}\right)$ all $\left(a_{1}^{2} a_{2}^{2} a_{3}^{2}\right)$ all $\left(a_{1}^{3} a_{2}^{3} a_{3}^{3}\right)$ all $\left(a_{1}^{4} a_{2}^{4} a_{3}^{4}\right)$ all $\left(a_{1}^{5} a_{2}^{5} a_{3}^{5}\right)$ all $=H_{7776}$,

II. $\left\{H_{7776}\right\}$ pos $=H_{3888}$,

III. $\left\{H_{7776}\right\}_{3,3,3,3,3}=H_{486}$,

IV. $\left(a_{1}^{1} a_{2}^{1} a_{3}^{1}\right) \operatorname{pos}\left(a_{1}^{2} a_{2}^{2} a_{3}^{2}\right) \operatorname{pos}\left(a_{1}^{3} a_{2}^{3} a_{3}^{3}\right) \operatorname{pos}\left(a_{1}^{4} a_{2}^{4} a_{3}^{4}\right) \operatorname{pos}\left(a_{1}^{5} a_{2}^{5} a_{3}^{5}\right) \operatorname{pos}=H_{243}$,

V. $\left(a_{1}^{1} a_{2}^{1} a_{3}^{1} \cdot a_{1}^{2} a_{2}^{2} a_{3}^{2} \cdot a_{1}^{3} a_{2}^{3} a_{3}^{3} \cdot a_{1}^{4} a_{2}^{4} a_{3}^{4} \cdot a_{1}^{5} a_{2}^{5} a_{3}^{5}\right)$ all $=I_{6}$,

VI. $\quad\left(a_{1}^{1} a_{2}^{1} a_{3}^{1} \cdot a_{1}^{2} a_{2}^{2} a_{3}^{2} \cdot a_{1}^{3} a_{2}^{3} a_{3}^{3} \cdot a_{1}^{4} a_{2}^{4} a_{3}^{4} \cdot a_{1}^{5} a_{2}^{5} a_{3}^{5}\right)$ сус $=H_{3}$,

VII. Unity.

Denoting the system with index $r$ by $A_{r}$, it is evident that these systems must be interchanged according to the five groups $\left(A_{1} A_{2} A_{3} A_{4} A_{5}\right)$ cyc, $\left(A_{1} A_{2} A_{3} A_{4} A_{5}\right)_{10}$, $\left(A_{1} A_{2} A_{3} A_{4} A_{5}\right)_{20}$, $\left(A_{1} A_{2} A_{3} A_{4} A_{5}\right)$ pos, $\left(A_{1} A_{2} A_{3} A_{4} A_{5}\right)$ all.

The order of procedure in each case is as follows:

1. If the group is to correspond to $\left(A_{1} A_{2} A_{3} A_{4} A_{5}\right)$ cyc, a substitution $s$ must be found that will interchange the systems cyclically, transform the head into itself, and have its fifth power in the head. The imprimitive group so generated may be called $G$.

2. If the group is to correspond to $\left(A_{1} A_{2} A_{3} A_{4} A_{5}\right)_{10}$, it must contain $G_{1}$ as a self-conjugate subgroup. In addition, therefore, to the $s$ of case 1, a substitution $t$ must be found that will interchange four of the systems in two pairs, as $A_{2} A_{5} \cdot A_{3} A_{4}$, while leaving the remaining system unaltered, and that will, at the same time, transform the head into itself and $G$ into itself. This substitution $t$ must also have its square in the head. This imprimitive group shall be called $G_{2}$.

3. If the group is to correspond to $\left(A_{1} A_{2} A_{3} A_{4} A_{5}\right)_{20}$, it must contain both $G_{1}$ and $G_{i z}$ as self-conjugate subgroups. In addition, therefore, to the $s$ of case 1 , a substitution $u$ must be found interchanging four of the systems cyclically, according to $A_{2} A_{3} A_{5} A_{4}$ for instance, transforming $G_{1}$ and $G_{2}$ in to themselves and having its fourth power in the head.

4. If the group is to correspond to $\left(A_{1} A_{2} A_{3} A_{4} A_{5}\right)$ pos, two substitutions, $v$ and $v^{\prime}$, must be found corresponding to $A_{1} A_{2} A_{3}$ and $A_{1} A_{4} A_{5}$. These sub- 
stitutions must, therefore, each interchange three systems, leaving two unaltered, they must have their cubes in the head, and must transform the head into itself. This group may be called $G^{\prime}$.

5. If the group is to correspond to $\left(A_{1} A_{2} A_{3} A_{4} A_{5}\right)$ all, two substitutions, $w$ and $w^{\prime}$, must be found corresponding to $A_{1} A_{2} A_{3} A_{4}$ and $A_{1} A_{5} . \quad G^{\prime}$ is to be contained in this new group as a self-conjugate subgroup, therefore $w$ and $v^{\prime}$ must transform the head into itself and $G^{\prime}$ into itself. The fourth power of $v$ and the square of $w^{\prime}$ must both be contained in the head.

I. $H_{7776}$ is the largest possible intransitive group with the given systems of intransitivity, and, consequently, only one group with this head corresponds to each of the transitive groups of degree 5. For each of these groups a substitution or pair of substitutions can be found fulfilling all required conditions and involving the elements of the systems symmetrically. A second set could be found only by multiplying this first step by some substitution belonging to the largest group that contains the head self-conjugately without interchanging any of the systems. But this group is the head itself. The required groups are, therefore, the following:

$$
\begin{aligned}
& \left\{H_{7776}, s\right\} \text { of order } 38880, \\
& \left\{H_{7776}, s, t\right\} \text { of order } 77760, \\
& \left\{H_{7776}, s, u\right\} \text { of order } 155520, \\
& \left\{I_{7776}, v, v^{\prime}\right\} \text { of order } 466560_{1}, \\
& \left\{H_{7776}, w, w^{\prime}\right\} \text { of order } 933120,
\end{aligned}
$$

where

$$
\begin{aligned}
& s=a_{1}^{1} a_{1}^{2} a_{1}^{3} a_{1}^{4} a_{1}^{5} \cdot a_{2}^{1} a_{2}^{2} a_{2}^{3} a_{2}^{4} a_{2}^{5} \cdot a_{3}^{1} a_{3}^{2} a_{3}^{3} a_{3}^{4} a_{3}^{5}, \\
& t=a_{1}^{2} a_{1}^{5} \cdot a_{1}^{3} a_{1}^{4} \cdot a_{2}^{2} a_{2}^{5} \cdot a_{2}^{3} a_{2}^{4} \cdot a_{3}^{3} a_{3}^{5} \cdot a_{3}^{3} a_{3}^{4} \text {, } \\
& u=a_{1}^{3} u_{1}^{3} u_{1}^{5} a_{1}^{4} \cdot a_{2}^{2} u_{2}^{3} a_{2}^{5} a_{2}^{4} \cdot a_{3}^{2} a_{3}^{3} u_{3}^{5} a_{3}^{4}, \\
& v=a_{1}^{1} a_{1}^{2} u_{1}^{3} \cdot c_{2}^{1} a_{2}^{2} a_{2}^{3} \cdot a_{3}^{1} a_{3}^{2} a_{3}^{3} \text {, } \\
& v^{\prime}=a_{1}^{1} a_{1}^{4} a_{1}^{5} \cdot a_{2}^{1} a_{2}^{4} a_{2}^{5} \cdot a_{3}^{1} a_{3}^{4} a_{3}^{5}, \\
& w=a_{1}^{1} a_{1}^{2} c_{1}^{3} a_{1}^{4} \cdot a_{2}^{1} c_{2}^{2} a_{2}^{3} a_{2}^{4} \cdot a_{3}^{1} a_{3}^{2} a_{3}^{3} a_{3}^{4}, \\
& w^{\prime}=a_{1}^{1} a_{1}^{5} \cdot a_{2}^{1} a_{2}^{5} \cdot a_{3}^{1} a_{3}^{5} \text {. }
\end{aligned}
$$

These letters shall be kept throughout this section of the paper to denote these symmetrically formed substitutions, other substitutions with corresponding properties being denoted by the same letter's with suffixes. 
II. $H_{3898}$ gives only one group isomorphic to $\left(A_{1} A_{2} A_{3} A_{1} A_{5}\right)$ cyc, viz., the group generated by $s$. Any new $s_{a}$ must have as cofactor an odd substitution belonging to $H_{77 \pi 6}$, but the fifth power of such a substitution is not contained in the head. There are, however, two groups isomorphic to $\left(A_{1} A_{2} A_{3} A_{4} A_{5}\right)_{10}$, since both $t$ and $t_{a}=a_{1}^{1} a_{2}^{1} . t$ fulfill the necessary conditions. The former generates a group $G_{38880_{2}}$ containing only even substitutions, the latter generates a group $G_{3380_{3}}$ containing both odd and even substitutions. There are likewise two groups isomorphic to $\left(A_{1} A_{2} A_{3} A_{4} A_{5}\right)_{20}$, one generated by $u$, the other by $a_{1}^{1} a_{2}^{1} \cdot u$. The first of these groups contains odd substitutions, the second only even. $G_{38802}$ is contained self-conjugately in both.

Only one group $G^{\prime}$ can be found for this head, as no new $v_{a}$ or $v_{a}^{\prime}$ fulfills the necessary conditions. Such a substitution would necessarily be of the form $\sigma v$ or $\sigma v^{\prime}$, where $\sigma$ would belong to the group $H_{i n i 6}$. If $\sigma$ were even, the group so generated would be a repetition of the group generated by $v$ and $v^{\prime}$. If $\sigma$ were odd, the cubes of $\sigma v, \sigma v^{\prime}$ would not be contained in the head.

Two groups can be found isomorphic to $\left(A_{1} A_{2} A_{3} A_{4} A_{5}\right)$ all, the substitutions $w$ and $w^{\prime}$ generating one group, the substitutions $a_{1}^{1} a_{2}^{1} \cdot w, a_{1}^{1} a_{2}^{1} \cdot w^{\prime}$ generating the other. This latter group contains only even substitutions.

From this head we have, therefore, derived eight groups:

$$
\begin{aligned}
& \left\{H_{3888}, s\right\} \text { of order } 19440, \\
& \left\{H_{3888}, s, t\right\} \text { of order } 38880_{2}, \\
& \left\{H_{3888}, s, a_{1}^{1} a_{2}^{1} \cdot t\right\} \text { of order } 38880_{3}, \\
& \left\{H_{3388}, s, u\right\} \text { of order } 76660_{2}, \\
& \left\{H_{3888}, s, a_{1}^{1} a_{2}^{1}, u\right\} \text { of order } 76660_{3}, \\
& \left\{H_{3888}, v, v^{\prime}\right\} \text { of order } 233280, \\
& \left\{H_{3888}, w, u^{\prime}\right\} \text { of order } 466560_{2}, \\
& \left\{H_{3888}, a_{1}^{1} a_{2}^{1}, u, a_{1}^{1} \alpha_{2}^{1} \cdot w^{\prime}\right\} \text { of order } 466560_{3} .
\end{aligned}
$$

11I. $H_{456}$ furnishes us with only one group isomorphic to $\left(A_{1} A_{2} A_{3} A_{4} A_{5}\right)$ cyc, for an examination of the groups given by all possible types of substitutions $s_{a}$ shows that each of these groups is merely the group generated by the help of $s$ and transformed with respect to some easily discovered substitution. Moreover, there is but one group isomorphic to $\left(A_{1} A_{2} A_{3} A_{4} A_{5}\right)_{10}$, viz., that generated with the help of $t$. Any cofactor of $t$ must be of one of the types $a_{1}^{1} a_{2}^{1}, a_{1}^{2} a_{2}^{2} . a_{1}^{5} a_{2}^{5}$, $a_{1}^{1} a_{2}^{1}, a_{1}^{2} a_{2}^{2} \cdot a_{1}^{5} a_{2}^{5}, a_{1}^{2} a_{2}^{2}, a_{1}^{3} a_{2}^{3} \cdot a_{1}^{4} a_{2}^{1} \cdot a_{1}^{5} a_{2}^{5}$, but any $t_{a}$ got by means of these, transforms 
$s$ into $s^{4}$ (a substitution not in the head). Precisely the same reasoning shows that there is only the one group isomorphic to $\left(A_{1} A_{2} A_{3} A_{1} A_{5}\right)_{20}$.

In addition to the group isomorphic to $\left(A_{1} A_{2} A_{3} A_{4} A_{5}\right)$ pos generated by means of the substitutions $v$ and $v^{\prime}$, we must examine groups generated with the help of $v_{a}$ and $v_{a}^{\prime}$, substitutions which contain as cofactors of $v, v^{\prime}$ respectively the products of transposition, one transposition from each system. $\Lambda$ number of these may be rejected at once, but we are left with the possible forms:

$$
\begin{aligned}
& v_{1}=a_{1}^{1} a_{2}^{1} \cdot a_{1}^{2} \alpha_{2}^{2} \cdot v=a_{1}^{1} \alpha_{2}^{2} \alpha_{1}^{3} \cdot a_{2}^{1} a_{1}^{2} \alpha_{2}^{3} \cdot a_{3}^{1} \epsilon_{3}^{2} \iota_{3}^{3}, \\
& v_{2}=a_{1}^{1} a_{2}^{1} \cdot a_{1}^{4} \tau_{2}^{4} \cdot a_{1}^{5} a_{2}^{5} \cdot v=a_{1}^{1} \tau_{2}^{2} a_{2}^{3} \cdot a_{2}^{1} \iota_{1}^{2} a_{1}^{3} \cdot a_{1}^{4} a_{2}^{4} \cdot a_{1}^{5} a_{2}^{5}, \\
& v_{1}^{\prime}=a_{1}^{1} a_{2}^{1} \cdot a_{1}^{4} a_{2}^{4} \cdot v^{\prime}=a_{1}^{1} e_{2}^{4} e_{1}^{5} \cdot a_{2}^{1} e_{1}^{4} a_{2}^{5} \cdot a_{3}^{1} a_{3}^{4} e_{3}^{5}, \\
& v_{2}^{\prime}=a_{1}^{1} a_{2}^{1} \cdot a_{1}^{3} a_{2}^{2} \cdot a_{1}^{3} a_{2}^{3} \cdot v^{\prime}=a_{1}^{1} a_{2}^{4} a_{2}^{5} \cdot a_{2}^{1} a_{1}^{4} a_{1}^{5} \cdot a_{1}^{2} a_{2}^{2} \cdot a_{1}^{3} a_{2}^{3} .
\end{aligned}
$$

But, since $v_{1}, v_{2}^{4}$ are transformable into $v$, and $v_{1}^{\prime}, v_{2}^{\prime 4}$ are transformable in to $v^{\prime}$, it is impossible to generate any group by means of any combination of these four substitutions excepting a group that can be transformed into the one gencrated by means of $v$ and $v^{\prime}$.

A similar examination of all groups isomorphic to $\left(A_{1} A_{2} A_{3} A_{4} A_{5}\right)$ all, shows that, in addition to the group generated with the help of $w$ and $w^{\prime}$, there is one other group generated by means of $w_{a}=a_{1}^{5} \alpha_{2}^{5} . w$ and $w^{\prime}$.

From this head are therefore formed the six following groups:

$$
\begin{aligned}
& \left\{I_{486}, s\right\} \text { of order } 2430_{1}, \\
& \left\{I_{486}, s, t\right\} \text { of order } 4860_{1}, \\
& \left\{I_{486}, s, u\right\} \text { of order } 9720, \\
& \left\{I_{486}, v, v^{\prime}\right\} \text { of order } 29160_{1}, \\
& \left\{I_{486}, w, w^{\prime}\right\} \text { of order } 58320_{1}, \\
& \left\{I_{436}, a_{1}^{5} \alpha_{2}^{5} \cdot w, w^{\prime}\right\} \text { of order } 58320_{2} .
\end{aligned}
$$

IV. $I I_{243}$ gives one group isomorphic to $\left(A_{1} A_{2} A_{3} A_{4} A_{5}\right)$ cyc, by means of $s$. The only other permissible forms of $s_{a}$ are of the type

$$
s_{1}=a_{1}^{1} \alpha_{2}^{1} \cdot a_{1}^{2} a_{2}^{2} \cdot s, \quad s_{2}=a_{1}^{1} \alpha_{2}^{1} \cdot a_{1}^{2} \alpha_{2}^{2} \cdot a_{1}^{3} a_{2}^{3} \cdot a_{1}^{4} \alpha_{2}^{4} \cdot s .
$$

But $s_{1}$ and $s_{2}$ are each the transformed of $s$ with respect to some substitution that transforms the head into itself; therefore, there is only the one group of this type. On the other hand, there are two groups isomorphic to $\left(A_{1} A_{2} A_{3} A_{4} A_{5}\right)_{10}$, since both $t$ and $t_{1}=a_{1}^{1} a_{2}^{1} \cdot a_{1}^{2} e_{2}^{2} \cdot a_{1}^{3} a_{2}^{3} \cdot a_{1}^{4} e_{2}^{1} \cdot a_{1}^{5} \alpha_{2}^{5} \cdot t$ fulfill all necessary conditions and generate, one a group of even substitutions, the other a group containing odd 
substitutions. There are also two groups isomorphic to $\left(A_{1} A_{2} A_{3} A_{4} A_{5}\right)_{20}$, one containing both odd and even substitutions, the other only even. These are generated respectively by means of $u$ and of $u_{1}=a_{1}^{1} a_{2}^{1} \cdot a_{1}^{2} u_{2}^{2} \cdot a_{1}^{3} a_{2}^{3} \cdot a_{1}^{4} a_{2}^{4} \cdot a_{1}^{5} a_{2}^{5} \cdot u$, and each contains as a self-conjugate subgroup the group isomorphic to $\left(A_{1} A_{2} A_{3} A_{4} A_{5}\right)_{10}$ that consists entirely of even substitutions.

Only one group can be found isomorphic to $\left(A_{1} A_{2} A_{3} A_{1} A_{5}\right)$ pos, and this is the one formed by the help of $v$ and $v^{\prime}$. An examination of the various substitutions $v_{a}$ and $v_{a}^{\prime}$ corresponding to various types of cofactor of $v$ and $v^{\prime}$ shows that all groups formed by means of these substitutions are transformable into the one group.

On the other hand, we have two distinct groups corresponding to $\left(A_{1} A_{2} A_{3} A_{4} A_{5}\right)$ all, the one consisting of both odd and even substitutions aud generated by the aid of $w$ and $w^{\prime}$, the other consisting entirely of even substitutions and generated by the aid of $a_{1}^{5} a_{2}^{5} \cdot w$ and $a_{1}^{2} a_{2}^{2} \cdot w^{\prime}$.

From this head we have, therefore, the eight following groups:

$$
\begin{aligned}
& \left\{H_{243}, s\right\} \text { of order } 1215, \\
& \left\{H_{243}, s, t\right\} \text { of order } 2430_{2}, \\
& \left\{H_{243}, s, a_{1}^{1} a_{2}^{1} \cdot a_{1}^{2} a_{2}^{2} \cdot a_{1}^{3} a_{2}^{3} \cdot a_{1}^{4} a_{2}^{4}, a_{1}^{5} a_{2}^{5} \cdot t_{;}^{\prime} \text { of order } 2430_{3},\right. \\
& \left\{H_{243}, s, u\right\} \text { of order } 4860_{2}, \\
& \left\{H_{243}, s, a_{1}^{1} a_{2}^{1} \cdot a_{1}^{2} a_{2}^{2} \cdot a_{1}^{3} a_{2}^{3} \cdot a_{1}^{4} a_{2}^{4}, a_{1}^{5} a_{2}^{5} \cdot u\right\} \text { of order } 4860_{3}, \\
& \left\{H_{243}, v, v^{\prime}\right\} \text { of order } 14580, \\
& \left\{H_{243}, u, w^{\prime}\right\} \text { of order } 29160_{2}, \\
& \left\{H_{243}, a_{1}^{5} a_{2}^{5} \cdot u, a_{1}^{2} a_{2}^{2} \cdot w^{\prime}\right\} \text { of order } 29160_{3} .
\end{aligned}
$$

V. $H_{6}$ furnishes one group corresponding to each transitive group of degree 5. These groups are generated respectively by the substitutions $s, t, u, v, v^{\prime}, w, v v^{\prime}$, and can readily be seen to be indentical with those of orders $30_{2}, 60_{2}, 120,360_{2}$, 720 included among the groups with three systems of imprimitivity. An interchange of suffixes and indices in the one set of groups gives the generating substitution of the other set of groups.

VI. $H_{3}$ furnishes groups corresponding to the transitive groups of degree 5 by means of the substitutions $s, t, u, v, v^{\prime}, w, w^{\prime}$. As in the last case, howerer, these correspond to the groups of orders $15,30_{1}, 60_{1}, 180,360_{1}$ included in the groups with three systems of imprimitivity. By the use of the cofactor $\sigma=a_{1}^{1} \alpha_{2}^{1}$. 
$a_{1}^{2} a_{2}^{2} \cdot a_{1}^{3} a_{2}^{3} \cdot a_{1}^{4} a_{2}^{4} \cdot a_{1}^{5} a_{2}^{5}$ three more groups can be found generated respeetively by the help of $t_{1}=\sigma t, u_{1}=\sigma u, w_{1}=\sigma v, w_{1}^{\prime}=\sigma v^{\prime}$. 'These groups, however, are seen to be identical with those of orders $30_{3}, 60_{3}$, and $360_{3}$ included in the groups with three systems of imprimitivity. This head gives, therefore, no group essentially new.

VII. In the discussion of the head unity a useful theorem is the following given by Frobenius (Crelle t. eI, p. 287):

The average number of elements in all the substitutions of a group is $n-\alpha, n$ being the degree of the group, and a the number of its trunsitive constituents.

'The only transitive groups of degree 5 containing 15 as a faetor of the order are the symmetric and altemating groups. We have therefore to find an imprimitive group of degree 15 with 5 systems of intransitivity simply isomorphic to the alternating (symmetrie) group in 5 letters.

In determining the imprimitive group eorresponding to $\left(A_{1} A_{2} A_{3} A_{4} A_{5}\right)$ pos, we make use of the following facts: (1) the 15 conjugate substitutions corresponding to terms of the type $A_{1} A_{2} . A_{3} A_{4}$ must be of degrees 12 or $14 ;(2)$ the 20 conjugate substitutions corresponding to terms of the type $A_{1} A_{2} A_{3}$ must be of degrees 9,12 , or 15 ; (3) the 24 eonjugate substitutions eorresponding to terms of the type $A_{1} A_{2} A_{3} A_{4} A_{5}$ must be of degree 15 . It must, therefore, be possible to solve the equation

$$
15(12+2 \alpha)+20(9+3 \beta)+24.15=14.60
$$

where $\alpha=0,1 ; \beta=0,1,2$. The only solution is $\alpha=0, \beta=2$.

Therefore the imprimitive group we are seeking contains among its substitutions 15 of degree 12 and order 2, 20 of degree 15 and order 3,24 of degree 15 and order 5. Making use of the relations among the generating substitutions of such a group of order 60 as given in Burnside, Theory of Groups, p. 107, we find that the two substitutions eorresponding to $A_{1} A_{2} A_{3} A_{4} A_{5}, A_{1} A_{2} A_{3} A_{4}^{7}$, substitutions which will generate $\left(A_{1} A_{2} A_{3} A_{4} A_{5}\right)$ pos, are respectively ,

$$
\begin{aligned}
& s=a_{1}^{1} a_{1}^{2} a_{1}^{3} a_{1}^{4} a_{1}^{5} \cdot a_{2}^{1} a_{2}^{2} a_{2}^{3} a_{2}^{4} a_{2}^{5} \cdot a_{3}^{1} a_{3}^{2} a_{3}^{3} c_{3}^{4} a_{3}^{5}, \\
& \rho=a_{1}^{1} a_{3}^{2} \cdot a_{1}^{3} a_{2}^{4} \cdot a_{2}^{1} a_{1}^{2} \cdot a_{2}^{3} a_{3}^{4} \cdot a_{3}^{1} a_{2}^{2} \cdot a_{3}^{3} a_{1}^{4} ;
\end{aligned}
$$

$s$ and $\rho$ are therefore the generating substitutions of an imprimitive group simply isomorphic to the alternating group of degree 5.

In determining a group simply isomorphic to $\left(A_{1} A_{2} A_{3} A_{4} A_{5}\right)$ all, we argue as before in regard to the various sets of conjugate substitutions. The 15 substitu- 
tions corresponding to terms of the type $A_{1} A_{2} . A_{3} A_{4}$ are of degrees 12 or 14 , the 20 corresponding to the type $A_{1} A_{2} A_{3}$ are of degrees 9,12 , or 15 , the 24 correspording to the type $A_{1} A_{2} A_{3} A_{4} A_{5}$ are of degree 15 , the 10 corresponding to the type $A_{1} A_{2}$ are of degrees $6,8,10$, or 12 ; the 30 corresponding to the type $A_{1} A_{2}$ $A_{3} A_{4}$ are of degrees 12 or 14 ; the 20 corresponding to the type $A_{1} A_{2} A_{3} A_{4} A_{5}$ are of degree 15. The equation to be satisfied is therefore

$$
\begin{aligned}
15(12+2 \alpha)+20 & (9+3 \beta)+24.15+10(6+2 \gamma)+30(12+2 \delta)+20.15 \\
& =14.120 \text { where } \alpha=0,1 ; \beta=0,1,2 ; \gamma=0,1,2,3 ; \delta=0,1 .
\end{aligned}
$$

The only solution is $\alpha=0, \beta=2, \gamma=3, \delta=1$. The substitutions $A_{1} A_{2} A_{3} A_{4} A_{5}$, $A_{2} A_{3} A_{4} A_{5}, A_{1} A_{2} A_{2} A_{4}$ will generate the group $\left(A_{1} A_{2} A_{3} A_{4} A_{5}\right)$ all, and corresponding to these as generators of the imprimitive group we have the three substitutions,

$$
\begin{aligned}
& s=a_{1}^{1} a_{1}^{3} a_{1}^{3} a_{1}^{4} a_{1}^{5} \cdot a_{2}^{1} a_{3}^{3} a_{2}^{3} a_{2}^{4} a_{2}^{5} \cdot a_{3}^{1} a_{3}^{2} a_{3}^{3} a_{3}^{4} a_{3}^{5}, \\
& \sigma=a_{2}^{1} a_{3}^{1} \cdot a_{1}^{7} a_{1}^{3} a_{1}^{5} a_{1}^{4} \cdot a_{2}^{2} a_{3}^{2} a_{2}^{5} a_{3}^{4} \cdot a_{3}^{2} a_{2}^{3} a_{3}^{5} a_{2}^{4} . \\
& \rho=a_{1}^{1} a_{3}^{2} \cdot a_{1}^{3} a_{2}^{4} \cdot a_{2}^{1} a_{1}^{2} \cdot a_{2}^{3} a_{3}^{4} \cdot a_{3}^{1} a_{2}^{2} \cdot a_{3}^{3} a_{1}^{4} .
\end{aligned}
$$

To sum up the results of the preceding work, the 16 heads with three systems of intransitivity give 41 groups with three systems of imprimitivity. The $i$ heads with five systems of intransitivity give 42 groups with five systems of imprimitivity, but of these 13 groups contain also three systems of imprimitivity. Therefore there are 70 imprimitive groups of degree 15 as determined in this paper.

\section{Primitive Substitution Groups of Degrce Eighteen.}

The main theorems employed in this investigation of prinitive groups are the following, in which $p$ is always to stand for a prime number.

I. The order of a primitive group of degree $n$ camnot excced $\frac{n !}{2.3 \ldots p}$, where $2,3, \ldots$ p are the distinct mimes which are less than $\frac{2}{3} n$. (Burnside, Theory of Groups, p. 199).

II. A group of degree $p+x$ or of degree $2 p+x, x>2$, connot be more than $x$ times transitive. (Miller, Bull. A. M. S., v. IV, pp. 142, 143).

III. If a jimitier group, of degree n contains a circular substitution of prime 
Fifteen and the Primitive Substitution Groups of Degree Eighteen.

order $p$, the group' is at least $(n-p+1)$-fold transitive. (Cole's tr. of Netto's Theory of Substitutions, p. 93).

IV. A self-conjugate subgroup of a primitive groupmest be transitive. (Burnside, l. c., p. 187).

V. A self-conjugate subgroup of a $x$-lly transitive group, of degree $n(2<x<n)$ is in general at leust $(x-1)$-ply transitive. The only excepition is that a triply transitive yroup of degree $2^{m}$ may have a self-conjugate subgroup of order $2^{m}$. (Burnside, l. c., p. 189).

VI. A group $G$ which is at leust doubly trunsitive either must be simple or mus contain a simple group $H$ as a self-conjugate subgroup. In the latter case no oprera tion of $G$ except identity is permatuble with every operation of $H$. The onlyexceptions to this statement are that a triply transitive group of degree $2^{m}$ may haie a self conjugate subgroup of order $2^{m}$, and that a doubly transitive group of deyree $p^{m}$ may have a self-conjugate subgroup of order $p^{m}$. (Burnside, l. c., p. 192).

VII. The substitutions of a transitive group $G$ which leave a given symbol unchanged form a maximal subyroup $G_{1}$, which is one of a set of $n$ conjugate subgroups, each leaving one of the nelements unaffected. (Burnside, 1. e., p. 140).

VIII. The number of substitutions of degree $l<n$ contuined in a transitive group of degree $n$ is equal to the number of sulstitutions of this sume degree l contained in the maxima'subgroup $G_{1}$ of degree $n-1$ multipliedby $\frac{n}{n-l}$. (Stated by Miller, Quar. Jour. of. Math. v. XXVIII, p. 215.)

IX. The average number of elements in all the substitutions of a group is $n-u$. $n$ being the degree of the group and a the number of its transitive constituents. (Frobenius, Crelle, t. Cr, p. 287.)

X. Sylow's theorem, as stated by Burnside, l. c., p. 92, or by Sylow, "Théorèmes sur les groupes de substitutions," Math. Ann., v. V (1872), pp. 584 et seq.

XI. The class of a primitive group of degree $n$ is the same as the cluss of its maximal subgroup that leaves one element unaffected. 
While the preceding theorems are used throughout the work on primitive groups, the following are used mainly in the determination of simply transitive primitive groups.

XII. A simply transitive primitive gronp $G$ of degree $n$ camot contain a transitive sulgroup of degree less than n. (Miller, Quar. Jour. of Math., v. XXVIII, p. 215.

XIII. When $G_{1}$ contains a self-conjugate subgroup $I$ of degree $n-\alpha, H$ must be intransitice, and it must be the transform with respect to substitutions of $G$ of any one of $a-1$ other subgroups of $G_{1}\left(H_{1}^{\prime}, H_{2}^{\prime}, \ldots H_{a-1}^{\prime}\right)$. (Miller, Proc. Lon. Math. Soc., v. XXVIII, p. 534.)

XIV. All the prime numbers uhich divide the order of one of the transitive constituents of $G_{1}$ divide also the orders of each of the other transitive constituents.

Corollary I. If one of the transitive constituents of $G_{1}$ is of a mime degree, each of its other transitive constituents is of the same or a larger degree, and the order of $G_{1}$ is the same as the order of the group formed by these other transitive constituents.

Corollary II. If the order of $G_{1}$ is not divisitile by the square of a prime number, all its transitive constituents are of the same order, and $G_{1}$ is formed by estublishing a simple isomorphism between them. (Miller, l. c., p. 536.)

$\mathrm{XV}$. If a transitive constituent of $G_{1}$ is of a mime order, the order of $G_{1}$ is the same prime number, and $G$ is of class $n-1$.

Corollary. If $G_{1}$ contains a constituent of degree 2 , its order is 2 , and the degree of $G$ is a prime number. (Miller, l. c., p. 536.)

The above theorems are given in the form and with the symbols most convenient for use, and so are not always exact quotations from the papers and books referred to, while the references given are not always references to the original paper in which the theorem appeared.

Applying these theorems now to the special case in which $n=18$, we proceed as follows:

Since $18=2.7+4$, by Theorem I a primitive group cannot be more than 4-ply transitive. 
By Theorem I the order is seen not to exceed

$$
\frac{18 !}{2 \cdot 3 \cdot 5 \cdot 7 \cdot 11}=2^{15} \cdot 3^{7} \cdot 5^{2} \cdot 7 \cdot 13 \cdot 17 \text {. }
$$

If the group included circular substitutions of orders $2,3,5,7,11,13$, it would be at least 17, 16,14,12,8,6-fold transitive respectively according to Theorem III. This is impossible; therefore circular substitutions of these orders are not present, and consequently we see at once that 11 and 13 cannot be factors of the order.

If the order includes the factor 7 , then, by Theorem X, there is a subgroup of order 7. This must consist of the powers of a substitution composed of two cycles of 7 elements each, and it must be contained self-conjugately in a group of order $7.4 \mathrm{~m}$ that interchanges transitively among themselves the four remaining elements. (Cf. Burnside, Theory of Groups, p. 202.) It is quite possible to establish a $(7 \alpha, 1)$ isomorphism between an imprimitive group of degree 14 with the systems of imprimitivity 7,7 and a transitive group of degree 4 ; therefore 7 may be a factor of the order.

A subgroup of order $5^{2}$ cannot be present, as it would have to be intransitive with the systems of intransitivity 5,5 or $5,5,5$. In the one case, it would have to be contained self-conjugately in a group of order $52.8 \mathrm{~m}$, in the other, in a group of order $5^{2} .3 . \mathrm{m}$. In either case, a circular substitution of order 5 would be present, which is impossible.

The factor 5 may be contained in the order, as it is possible to establish a $(5,1)$ isomorphism between the cyclical group of degree 15 and the cyclical group in the remaining three letters.

The order must, therefore, be a factor of $2^{15} \cdot 3^{7} \cdot 5 \cdot 7 \cdot 17$.

\section{Simply Trunsitive Groups.}

The maximal subgroup $G_{1}$ that leaves $a_{1}$ unaffected is intransitive (Theorem XII), and its order is, therefore, a factor of $2^{14} \cdot 3^{5} \cdot 5 \cdot 7$. Moreover, its class cannot be less than 6 , for if it were 2,3 or $5, G_{1}$ would necessarily contain a transitive subgroup of too low a degree, and it cannot be of elass 4 , if $G$ is to be primitive. (Netto, l. c., p. 138.)

By Theorem XIV, Cor. I, it is evident that $G_{1}$ cannot contain a transitive constituent of degree 13 or 11 ; by Theorem XIV it cannot contain a transitive 
constituent of degree 15 or 14 , and by Theorem XV, Cor., it cannot contain a constituent of degree 2 .

If one of the transitive constituents of $G_{1}$ is of degree 12 , the other must be of degree 5. The isomorphism between the transitive groups of degrees 12 and 5 must be an $(\alpha, 1)$ isomorphism, where $\alpha$ itself may be equal to 1 . By Theorem XIV, the order of the group of degree 5 must contain the factors $5,3,2$; therefore this group must be either the alternating or the symmetric group of degree 5. If the isomorphism is more than simple, then the group of degree 12 must be an imprimitive group with 6 systems of imprimitivity. The head for such an imprimitive group as we require is the intransitive group of order 2 and degree 12 given by $\left[1, a_{1} a_{2}, a_{3} a_{4}, a_{5} a_{6}, a_{7} a_{8}, a_{9} a_{10}, a_{11} a_{12}\right]$. The group $\left(a_{13} a_{14} a_{15} a_{16} a_{17}\right)$ pos contains

24 conjugate substitutions of order 5 and degree 5 , 20 conjugate substitutions of order 3 and degree 3 , 15 conjugate substitutions of order 2 and degree 4.

Corresponding to these in the group of degree 17, we have 1 substitution of degree 12 and order 2, 24 of degree $15+2 \alpha$ and order 5 or 10, 24 others of degree $15+2 \alpha^{\prime}$ and order 5 or 10,20 of degree $15+2 \beta$ and order 3 or 6 , ogether with 20 of degree $15+2 \beta^{\prime}$ and order 3 or 6,15 of degree $12+2 \gamma$ and order 2 or 4 , and 15 of degree $12+2 \gamma^{\prime}$ and order 2 or 4 , where

$$
\alpha=0,1 ; \alpha^{\prime}=0,1 ; \beta=0,1 ; \beta^{\prime}=0,1 ; \gamma=0,1,2 ; \gamma^{\prime}=0,1,2 .
$$

By Theorem IX, the following equation must be satisfied :

$$
\begin{aligned}
12+24(15+2 \alpha)+24\left(15+2 \alpha^{\prime}\right) & +20(15+2 \beta)+20\left(15+2 \beta^{\prime}\right) \\
& +15(12+2 \gamma)+15\left(12+2 \gamma^{\prime}\right)=120.15 .
\end{aligned}
$$

The only type of solution is given by $\alpha=\beta=\gamma=\beta^{\prime}=0, a^{\prime}=1, \gamma^{\prime}=2 . G_{1}$, therefore, contains both a self-conjugate subgroup of degree 12 and order 2 , and 15 conjugate subgroups of the same type. But, by Theorem XIII, only 5 such conjugate subgroups should exist if this group is to be the $G_{1}$ of a simply transitive primitive group. This intransitive group gives us, therefore, no such group as we require.

For precisely the same reason the intransitive group formed by establishing a $(2,1)$ isomorphism between an imprimitive group of degree 12 and order 240 , 
and the symmetric group of degree 5 cannot be employed in the formation of a simply transitive primitive group of degree 18 .

If the isomorphism is simple, the group $G_{1}$, including the alternating group of degree 5, must contain 24 substitutions of degree 10 or 15 and order 5,20 of degree $6,9,12$ or 15 and order 3,15 of degree $6,8,10,12,14$ or 16 and order 2. The following equation must, therefore, be satisfied: $24(10+\alpha)+20(6+\beta)$ $+15(6+\gamma)=60 \times 15$, where $\alpha=0,5 ; \beta=0,3,6,9 ; \gamma=0,2,4,6,8,10$. The only solution is $\alpha=5, \beta=9, \gamma=10 . G_{1}$ is, therefore, of class 15 .

A group $G$ of degree 18, formed with the help of this $G_{1}$ and, therefore, of order 60.18 , contains 36 conjugate subgroups of order 5 , each of which is contained self-conjugately in a group of order 30. As each of these subgroups of order 5 is already self-conjugate in a group of order 10, the construction of the generating substitutions of such a group is an easy matter. $G_{1}$ is generated by

and by

$$
s=a_{1} a_{3} a_{7} a_{5} a_{9} \cdot a_{2} a_{4} a_{8} a_{6} a_{10} \cdot a_{13} a_{14} a_{15} a_{16} a_{17}
$$

and contains, as one of the above-mentioned groups of order 10 , the group generated by

$$
\begin{aligned}
& u=s t=a_{3} a_{8} a_{11} a_{6} a_{9} \cdot a_{1} a_{7} a_{12} a_{5} a_{10} \cdot a_{13} a_{17} a_{15} a_{16} a_{14}, \\
& v=s^{-1} t s=a_{1} a_{2} \cdot a_{3} a_{7} \cdot a_{4} a_{8} \cdot a_{5} a_{6} \cdot a_{4} a_{12} \cdot a_{10} t_{11} \cdot a_{13} a_{15} \cdot a_{14} a_{16} .
\end{aligned}
$$

The group $\{u, v\}$ is a subgroup of a group of degree 18 and order 30 formed by establishing a $(5,1)$ isomorphism between an imprimitive group of degree 15 and order 30 with $u$ and its powers as head and the symmetric group in the three elements $a_{1} a_{2} a_{18}$. The question then reduces to that of the determination of a substitution of degree 18 and order 3 that will transform the head $\{u\}$ into itself, interchange cyclically the three systems of $\{u\}$, and be in its turn transformed into its square by $v$. An examination of all substitutions fulfilling these conditions results in finding none that do not give, when combined with other substitutions of $G_{1}$, substitutions that cannot possibly belong to a simply transitive primitive group containing $G_{1}$ as a maximal subgroup.

There is no primitive or imprimitive group of degree 12 simply isomorphic to the group (abcdef $)_{120}$; consequently, no isomorphism can be established between the symmetric group of degree 5 and a transitive group of degree 12 .

There remains the question whether the symmetrie group of degree 5 ean be put in a simply isomorphic relation to one of the imprimitive groups of degree 
12 and order 120 that have both six and two systems of imprivitivity. Such groups of degree 12, however, contain two self-conjugate subgroups of orders 2 and 60 respectively, and, therefore, are not in a simply isomorphic relation to the symmetric group of degree 5.

If one of the transitive constituents is of degree 10 , the other can only be of degree 7. By Theorem XIV, the group of degree 10 must contain 7 as a factor of its order, and, therefore, must be either the alternating or the symmetrie group. It is impossible to establish an isomorphic relation between either of these groups and one of degree 7 without introducing substitutions of too low a degree.

If one of the transitive constituents is of degree 9, the remaining constituent may be either intransitive in two systems of four elements each or intransitive in eight elements. The isomorphism can in neither case be simple, as an examination of all groups of degree $s$ and orders equal to those of transitive groups of degree 9 shows that in each case a system of intransitivity of degree 2 enters, with the single exception of a group of order 144. Here, however, the group of degree 9 contains a substitution of order 8 , while an inspection of the corresponding groups of degree 8 shows no substitution of that order.

The isomorphism is, therefore, an $(\alpha, \beta)$ isomorphism, where $\alpha$ and $\beta$ are not simultaneously equal to one.

When neither $\alpha$ nor $\beta$ is equal to one, $G_{1}$ must be formed from an imprimitive group of degree 9 and an intransitive group of degree 8 . The order of each transitive constituent must contain 3 as a factor, and, therefore, the group of degree 8 must be some combination of the alternating and symmetric groups of degree 4 in two systems of elements. The only combinations possible, consistent with the requirements of class, are got by establishing a simple isomorphism between the two symmetric groups of degree 4 or between the two alternating groups of the same degree. Every relation of isomorphism established between these groups of degree 8 and any imprimitive groups of degree 9 consistent with the requirements of class, results in a $G_{1}$ that contains a self-conjugate subgroup of order 4 and degree 8 , and no other subgroups of the same order. This case, therefore, gives no simply transitive primitive group.

When a becomes 1, the group of degree 8 must, as before, be composed of either the symmetric or the alternating groups of degree 4 in two sets of elements put into the relation of simple isomorphism. The order of such a group does not contain 9 as a factor; therefore this case gives no possible $G_{1}$. 
When $\beta$ becomes 1 , the group of degree 9 must be imprimitive. No transitive group of degree 8 stands, however, in the given relation of isomorphism towards an imprimitive group of degree 9 . The only permissible intransitive groups of degree 8 are combinations of the symmetric and alternating groups of degree 4 in two sets of elements, and none of these are isomorphic in the given way to any imprimitive group of degree 9.

If one of the transitive constituents is of degree 8 , we may have the systems 8 , 6,3 or $8,3,3,3$. In both cases we have an $(\alpha, 1)$ isomorphism between an intransitive group of degree 14 and the symmetric group of degree 3 . The group of degree 8 is not primitive, as no suitable isomorphic relation can be established between a primitive group of degree 8 and an imprimitive or an intransitive group of degree 6 . The only imprimitive groups of degree 8 that can be used are those with the head $\left(1, a_{1} a_{2} \cdot a_{3} a_{4} \cdot a_{5} a_{6} \cdot a_{7} a_{8}\right)$ that are isomorphic to a group of degree 4 and order 12 or 24 . Such groups, however, cannot be combined with the groups in the remaining 9 elements in such a way as to generate a group capable of being the $G_{1}$ of one of the required primitive groups.

The case in which $G_{1}$ contains a transitive constituent of degree 7 has already been discussed, as according to Theorem XIV, Cor. I, the remaining constituents must be of larger degree.

If $G_{1}$ contains a transitive constituent of degree 6 , the systems may be either $6,6,5$ or $6,4,4,3$. For the former system the only possible arrangement is to establish a simple isomorphism between the three groups $\left(a_{1} a_{2} a_{3} a_{4} a_{5} a_{6}\right)_{60}, \quad\left(a_{7} a_{8} a_{4} a_{30}\right.$ $\left.a_{11} a_{12}\right)_{60},\left(a_{13} a_{14} a_{15} a_{16} a_{17}\right)$ pos, or between the groups $\left(a_{1} a_{3} a_{3} a_{4} a_{5} a_{6}\right)_{120},\left(a_{7} a_{8} a_{9} a_{10} a_{11} a_{12}\right)_{120}$ $\left(a_{13} a_{14} a_{15} a_{16} a_{17}\right)$ all. An examination of the two groups $G_{1}$ formed from these isomorphisms shows that these are not the maximal subgroups of simply transitive primitive groups of degree 18 .

If the systems are $6,4,4,3$, only the imprimitive groups of degree 6 , the alternating and symmetric groups of degree 4, and the symmetric groups of degree 3 are involved. The group formed by the system $6,4,4$ has an $(\alpha, 1)$ isomorphism to the group of degree 3 , and this isomorphism cannot be simple. No combination of these groups can be found fulfilling all the necessary conditions.

The case in which $G_{1}$ contains a transitive system of degree 5 has already been discussed, as the remaining systems must be of degree greater than 5 .

If $G_{1}$ contains a transitive system of degree 4 , the only arrangement possible is $4,4,3,3,3$. 'The groups involved are therefore the symmetric groups of degree 3 and 4 , and the alternating group of degree 4. One group consistent with the 
requirements of class is got by establishing a $(1,4)$ isomorphism between the group,

$$
\left(a_{9} a_{10} a_{11} \cdot a_{12} a_{13} a_{14} \cdot a_{15} a_{16} a_{17}\right) \text { all, and }\left(a_{1} a_{2} a_{3} a_{4} \cdot a_{51} a_{6} a_{7} a_{8}\right) \text { all. }
$$

This group contains, howerer, one and only one subgroup of degree 8 and order 2.

A second group is got by lirst establishing a $(4,1)$ isomorphism between $\left(a_{1} a_{2} a\right.$ $\left.a_{4} \cdot a_{5} a_{6} a_{7} a_{8}\right)$ all and $\left(a_{9} a_{10} a_{11}\right)$ all; and then establishing a $(12,3)$ isomorphism between the group of order 24 so formed and the group $\left(a_{12} a_{13} a_{14} \cdot a_{15} a_{16} a_{17}\right)$ all. This group of degree 17 contains only one subgroup of order 4 and degree 8 ; therefore it cannot become a $G_{1}$.

$G_{1}$ cannot contain only systems of degree less than four, as in such a case a system of desree 2 would have to enter.

There is, therefore, no simply transitive primitive group of degree 18 . This result when joined to all other determinations of similar groups shows that there is no simply transitive primitive group of degree $p+1, p$ a prime number and $\leq 17$.

\section{Multiply transitive groups.}

Among the transitive groups of degree 17 five contain a self-conjugate subgroup of order 17. These are of order $17,2.17,4.17,8.17,16.17$ respectively, while all excepting the first are of class 16 .

If a primitive group of degree 18 and order 18.17 existerl, such a group would contain 18 conjugate subgroups of degree 17 . It would therefore contain 17 substitutions of degree 18 and 18.16 of degree 17. By Sylow's theorem since 18.17 $=2.3^{\circ}$. 17 , such a group contains either 1 or 34 subgroups of order $3^{2}$. A subgroup of this order must be intransitive, therefore cannot be self conjugate, and it is impossible to form 34 subgroups of order 9 from 17 substitutions of degree 18 . No such group of degree 18 exists.

A primitive group of degree 18 and order $18.17 .2=2^{2} .3^{2} .17$ would contain among its substitutions 153 of class 16 and order 2,288 of class 17 and order 17 , 170 of class 18. This group must contain either 1, 4, or 34 conjugate subgroups of order $3 \%$. As before, a subgroup of this order cannot be self-conjugate, as it is intransitive. If there were 4 conjugate subgroups, each would be selfconjugate in a group of order $3^{2} .17$ involving all 18 letters and necessarily transitive. Such a group is non-existent. If there were 34 conjugate subgroups they must be of degree 18 , and there are not enough substitutions of class 18 to form all these subgroups. 
A primitive group of degree 18 and order $18.17 .4=2^{3} .3^{2} .17$ contains among its substitutions 476 of degree 18, 459 of degree 16, 288 of degree 17. According to Sylow's theorem it contains either 1, 3, 9, 17, 51, or 153 conjugate subgroups of order $2^{3}$. Now the group leaving one element unchanged contains 17 conjugate subgroups of degree 16 and order 4 ; therefore the group of degree 18 contains 153 distinct conjugate subgroups of order 4 ; therefore it contains 153 conjugate subgroups of order $2^{3}$. Each of these is contained self-conjugately in no larger group.

The number of systems of intransitivity in any one is got from the following equation, where $x$ denotes the number of substitutions of degree 18 and $a$ the number of systems:

$$
18 x+16(7-x)=8(18-a), \text { where } a \neq 1, x<8 .
$$

There are $\mathrm{t} w 0$ sets of solutions, either $x=0, \alpha=4$, or $x=4, \alpha=3$.

The group of degree 17 is generated by,

$$
\begin{aligned}
& s=a_{1} a_{2} a_{3} a_{4} a_{5} a_{6} a_{7} a_{8} a_{9} a_{9} a_{10} a_{11} a_{12} a_{13} a_{14} a_{15} a_{16} a_{17} \\
& t=a_{2} a_{14} a_{17} a_{5} . a_{3} a_{10} a_{16} a_{9} \cdot a_{1} a_{6} a_{15} a_{13} \cdot a_{7} \ell_{11} \iota_{12} e_{8} \text {, }
\end{aligned}
$$

where $t$ and its powers form a self-conjugate subgroup of the group of order $2^{3}$ and degree 18 that is now under discussion. It is impossible to so connect the systems and introduce the remaining elements that the first solution may give the group of order $2^{3}$. Making use of the second solution we have only to combine with the group generated by $t$ a substitution of degree 18 that connects the two remaining elements by a transposition, and unites the cycles of $t$ in pairs. The 153 groups of order $2^{3}$ give in this way $153.4=612$ distinct substitutions of degree 18 , while there are only 476 in the group. This group of degree 18 does not exist.

If there is a primitive group of order $18 \cdot 17 \cdot 2^{3}=2^{4} \cdot 3^{2} \cdot 17$, it contains 288 substitutions of degree 17, 1071 of degree 16,1088 of degree 18. The group of degree 17 which is generated by

and

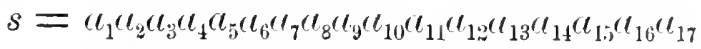

$$
\begin{aligned}
& t=a_{2} a_{10} a_{14} a_{16} a_{17} a_{19} a_{5} a_{3} \cdot a_{4} a_{11} a_{6} a_{12} a_{15} a_{8} a_{13} a_{7},
\end{aligned}
$$

contains 17 conjugate subgroups of degree 16 and order 8 ; therefore in the group of degree 18 there are 153 such conjugate subgroups, and each of these is self-conjugate in a group of order $2^{4}$ and degree 18 . Denoting by $\alpha$ the number of systems of intransitivity of this group of order $2^{4}$, and letting $x$ denote the number of substitutions of degree 18 contained in the group, we have the equation $18 x+16(15-x)=16(18-\alpha)$, where $\alpha \neq 1$. There are two solutions, $x=8$, $\alpha=2 ; x=0, \alpha=3$. The first solution would involve a larger number of substi- 
tutions of degree 18 than are actually present in the group under consideration. The second solution shows that the group must contain 153 conjugate subgroups of order $2^{4}$ and degree 18 consisting of substitutions of class $16 \mathrm{only}$, and involving three systems of intransitivity. A substitution must therefore be combined with $t$ that transforms $t$ into one of its powers. and has its head in the group generated l) $t$; moreover, this substitution must have as one of its cycles the transposition $\left(a_{1}\left(l_{18}\right)\right.$, and must have systems of intrinsitivity apart from this cycle consistent with the systems of $t$. Such a substitution is $\sigma=a_{2} a_{10} \cdot a_{3} a_{14} \cdot a_{9} a_{17} \cdot a_{5} a_{16} \cdot a_{6} a_{13} \cdot a_{7} a_{11}$. $a_{8} a_{12} \cdot a_{1} a_{13}$. The required group is therefore $\{s, t, \sigma\}$.

It is not necessary to prove that these three substitutions give a group of the required order, as such a group would be necessarily doubly transitive, and it is known that there is a doubly transitive group of degree 18 and of the required order. By the mode of construction of the substitutions, it is evident that there is only the one type of group of this degree and order.

Any primitive group of degree 18 and order 18.17 .16 contains 2312 substitutions of degree 18,288 of degree 17, 2295 of legree 16 . The group of degree 17 contains 17 conjugate cyclical subgroups of degree 16 and order 16 , therefore, the group of degree 18 contains 153 subgroups of order 16 , each of which is self-conjugate in one of 153 conjugate subgroups of order 32 . Giving $\alpha$ and $x$ the usual meanings, we find that the group of order 32 involves the equation $18 x+16(31-x)=32(18-\alpha)$, where $\alpha \neq 1$. The only solution is $\alpha=2$, $x=8$; therefore, the group of degree 12 and orler 32 must be intransitive with two systems of intransitivity, and must contain $S$ substitutions of degree 18, 23 of degree 16 . We have to add, therefore, to the cyclical group of degree $16, s$ substitutions of degree 16 and $s$ of degree 18 , all of them containing as one cycle the transposition of the remaining two letters.

The group of degree 17 and order 17.16 has as generators

and

$$
\begin{aligned}
& s=a_{1} a_{2} a_{3} a_{4} a_{5} a_{6} a_{7} a_{8} a_{9} l_{1} \cdot a_{11} l_{12} a_{13} a_{15} a_{15} l_{16} l_{17}
\end{aligned}
$$

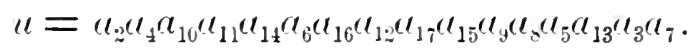

The substitution $\tau=a_{1} a_{18} \cdot a_{4}\left(\ell_{7} \cdot a_{3} a_{10} \cdot a_{5} \ell_{11} \cdot a_{6} \iota_{8} \cdot a_{9} \ell_{16} \cdot a_{13} a_{13} \cdot a_{12} \ell_{15}\right.$ generates with $s$ and $u$ the required group of degree 18 and order 17.16.18. A triply transitive group of such an order is known to exist (Burnside, l. c., p. 158); so no further proof that $\{s, u, \tau\}$ is a group is necessary. It is easy to see that the even substitutions of the group just found form the simple group of order 18.17 .8 . 
The three remaining transitive groups of degree 17 each contains 120 conjugate subgroups of order 17 . They are of orders $15.16 .17,15.16 .17 .2$, 15.16 .17 .4 respectively.

The group of degree 18 and order 15.16.17.18 would necessarily contain 816 conjugate subgroups of order 5 . Each is self-conjugate in a group of order 90 connecting the remaining three elements transitively. This group is intransitive with two transitive constituents, one of degree 15 and order 90 , the other of degree 3. The first, however, is non-existent, therefore, the group of degree 18 is non-existent.

The two remaining groups also, if they can generate primitive groups of degree 18, would generate groups that each contain 816 conjugate subgroups of order 5. In the one case, we should have to make use of an intransitive group containing as a transitive constituent a group of degree 15 and order 180, in the other, the transitive constituent would enter as a group of degree 15 and order 360. Both of these groups are non-existent; therefore, the three groups of degree 17, at present under discussion, furnish us with no new groups of degree 18.

As the case now stands, the conclusion arrived at may be summed up as follows :

There are no simply transitive primitive groups of degree 18, and in addition to the symmetric and alternating groups, there are only two multiply transitive groups of this degree, viz., the two given by

$$
\begin{aligned}
& \left\{\begin{array}{l}
\left(a_{1} a_{2} a_{3} a_{4} a_{5} a_{6} a_{7} a_{8} a_{9} a_{10} a_{11} a_{12} a_{13} a_{11} a_{15} a_{16} a_{17}\right), \\
\left(a_{2} a_{10} a_{14} a_{16} a_{17} a_{9} a_{5} a_{3} \cdot a_{4} a_{11} a_{6} a_{12} a_{15} a_{8} a_{13} a_{7}\right), \\
\left(a_{2} a_{10} \cdot a_{3} a_{14} \cdot a_{9} a_{17} \cdot a_{5} a_{16} \cdot a_{6} a_{13} \cdot a_{7} a_{11} \cdot a_{8} a_{12} \cdot a_{1} a_{18}\right),
\end{array}\right\} \text { of order } 2448, \\
& \left\{\begin{array}{l}
\left(a_{1} a_{2} a_{3} a_{4} a_{5} a_{6} a_{7} a_{8} a_{9} a_{10} a_{11} a_{12} a_{13} a_{11} a_{15} a_{16} a_{17}\right), \\
\left(a_{2} a_{4} a_{10} a_{11} a_{14} a_{6} a_{16} a_{12} a_{17} a_{15} a_{9} a_{8} a_{5} a_{13} a_{3} a_{7}\right), \\
\left(a_{1} a_{18} \cdot a_{1} a_{7} \cdot a_{3} a_{10} \cdot a_{5} a_{14} \cdot a_{6} a_{8} \cdot a_{9} a_{16} \cdot a_{11} a_{13} \cdot a_{12} a_{15}\right),
\end{array}\right\} \text { of order } 4896 .
\end{aligned}
$$

The second of these is triply transitive, and contains the first, which is doubly transitive and simple, as a self-conjugate subgroup.

The works consulted in the preparation of this paper have included, in addition to the standard works on the subject by Jordan, Serret, Netto, and Burnside, the following papers :

Askwith, "On Possible Groups of Substitutions that can be formed with 3, 4, 5, 6, 7 Letters Respectively." Quar. Jour. Math., v. XXIV (1890), pp. 111-167. 
Martin: On the Imprimitive Sulstitution Groups of Degree, etc.

"On Groups of Substitutions that can be formed with Eight Letters." Quar. Jour. Math., v. XXIV (1890), pp. 263-331.

"On Groups of Substitutions that can be formed with Nine Letters." Quar. Jour. Math., v. XXVI (1892), pp. 79-128.

Cayley, "On Substitution Groups for Two, Three, Four, Five, Six, Seven, and Eight Letters." Quar. Jour. Math., v. XXV (1891), pp. 71-88, 137-155.

Cole, "List of the Substitution Groups of Nine Letters." Quar. Jour. Math., v. XXVI (1892), pp. 372-388.

"The Transitive Substitution Groups of Nine Letters." Bull. Ner York Math. Soc., v. II (1893), pp. 250-258.

"List of the Transitive Substitution Groups of Ten and of Eleven Letters." Quar. Jour. Math., v. XXVII (1894), pp. 39-50.

"Note on the Substitution Groups of Six, Seven, and Eight Letters." Bull. New York Math. Soc., v. II (1893), pp. 184-190.

Miller, "Intransitive Substitution Groups of Ten Letters." Quar. Jour. Matb., v. XXVII (1894), pp. 99-118.

"List of Transitive Substitution Groups of Degree Twelve." Quar. Jour. Math., v. XXVIII (1896), pp. 193-231.

"Note on the Transitive Substitution Groups of Degree Twelve." Bull. Amer. Math. Soc., v. I (1894-1895), pp. 255-258.

"On the Transitive Substitution Groups of Degrees Thirteen and Fourteen." Quar. Jour. Math., v. XXIX (1898), pp. 224-249.

"On the Primitive Substitution Groups of Degree Fifteen." Proc. Lon. Math. Soc., v. XXVIII (1896-1897), pp. 533-544.

"On the Primitive Substitution Groups of Degree Sixteen." Amer. Jour. Math., v. XX (1898), pp. 229-241.

"On the Transitive Substitution Groups of Degree Seventeen." Quar. Jour. Math., v. XXXI (1899), pp. 49-57.

"A Simple Proof a Fundamental Theorem of Substitution Groups, and Several Applications of the Theorem." Bull. Amer. Math. Soc., v. II (18951896), pp. $75-77$.

"On the Limit of Transitivity of the Multiply Transitive Substitution Groups that do not Contain the Alternating Group." Bull. Amer. Math. Soc., v. IV (1897-1898), pp. 140-143.

Jordan, "Sur la classification des groupes primitifs." Comptes Rendus, t. LXXIII (1871), pp. 853-857.

"Sur l"énumeration des groupes primitifs pour les dix-sept premiers degrés." Comptes Rendus, t. LXXV (1872), pp. 1754-1757.

Philadelphia, Jamutay 1901. 


\section{LIFE.}

I was born in Elizabeth, New Jersey, December 30, 1869. In 1890, I entered Bryn Mawr College, selecting, as my major studies, Mathematics and Latin. In 1894, I received the degree of A. B. from this college. The first semester of the following year, 1894-1895, was spent at Bryn Mawr College as a graduate student in Mathematics and Physics, the second semester, in teaching at a preparatory school. During the year 1895-1896 I held the Fellowship in Mathematics in Bryn Mawr College, remaining there the following year, 18961897, as a graduate student. During the year 1897-1898 I held the Mary E. Garrett European Fellowship from Bryn Mawr College, and spent the entire year at the University of Göttingen, where I attended the lectures of Professors Klein and Hilbert. I then returned to Bryn Mawr College, where I was Fellow by Courtesy in Mathematics during the year 1898-1899. In the spring of $1899 \mathrm{I}$ passed the examination at Bryn Mawr College for the degree of Doctor of Philosophy. My major subject was Mathematics, pursued under the direction of Professors Scott and Harkness, while my double minor was Physics, pursued under the direction of Professor Mackenzie.

My gratitude is due to all the Professors under whom I have studied, and especially to Professor Harkness, under whose direction this paper was prepared. 
-

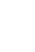

列

.

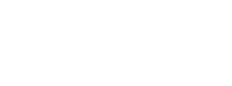

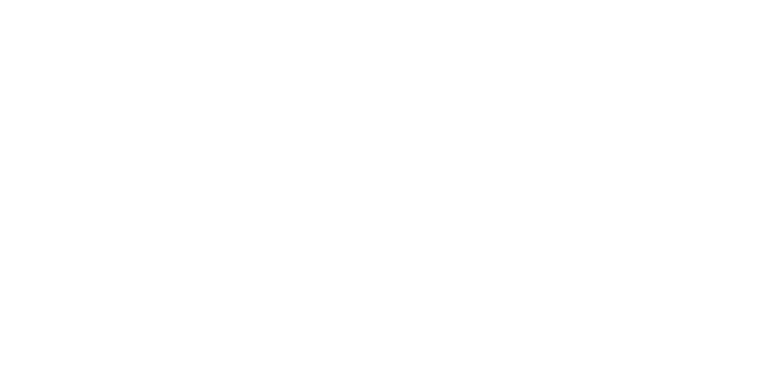

. 


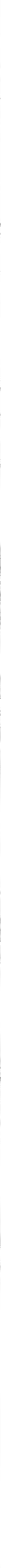


University of California

SOUTHERN REGIONAL LIBRARY FACILITY

405 Hilgard Avenue, Los Angeles, CA 90024-1388

Return this material to the library

from which it was borrowed.

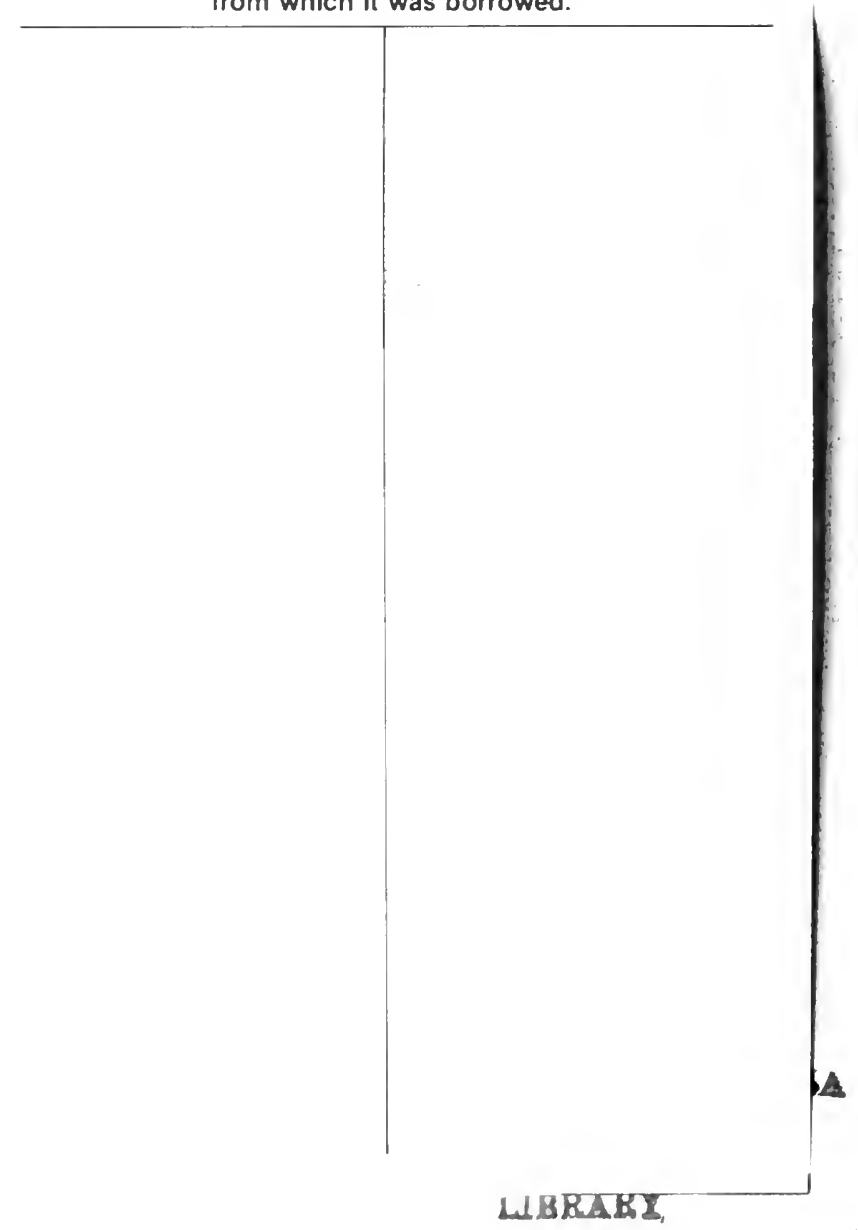




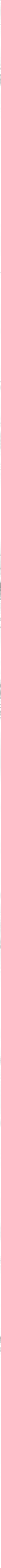

$$
\begin{aligned}
& \text { H. } 2^{2}-n^{2}+y^{2}
\end{aligned}
$$

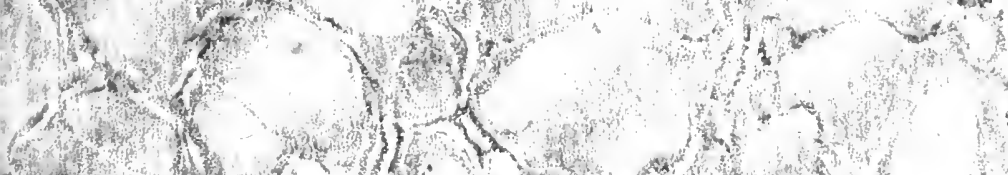

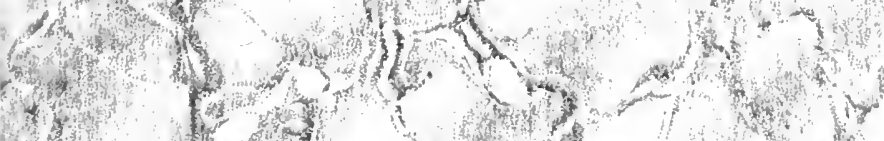




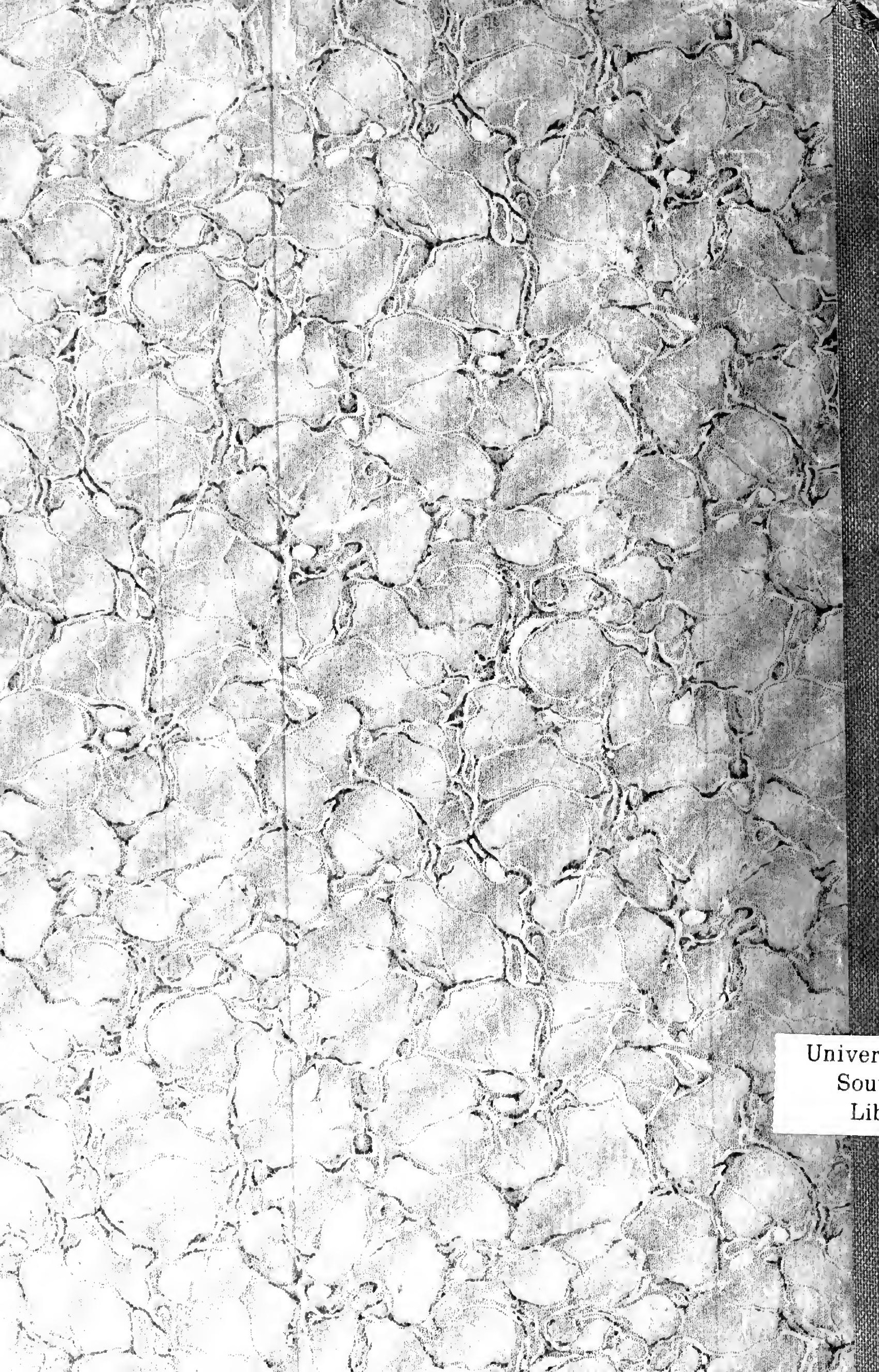

